\title{
The VMC Survey. XI. Radial Stellar Population Gradients in the Galactic Globular Cluster 47 Tucanae
}

\author{
Chengyuan Li $^{1,2,3}$, Richard de Grijs ${ }^{1,2}$, Licai Deng ${ }^{3}$, Stefano Rubele ${ }^{4}$, Chuchu Wang ${ }^{2}$, Kenji \\ Bekki $^{5}$, Maria-Rosa L. Cioni ${ }^{6,7}$, Gisella Clementini ${ }^{8}$, Jim Emerson ${ }^{9}$, Bi-Qing For ${ }^{5}$, Leo \\ Girardi $^{4}$, Martin A. T. Groenewegen ${ }^{10}$, Roald Guandalini ${ }^{11}$, Marco Gullieuszik ${ }^{4}$, Marcella \\ Marconi $^{12}$, Andrés E. Piatti ${ }^{13,14}$, Vincenzo Ripepi ${ }^{12}$, and Jacco Th. van Loon ${ }^{15}$
}

\begin{abstract}
We present a deep near-infrared color-magnitude diagram of the Galactic globular cluster 47 Tucanae, obtained with the Visible and Infrared Survey Telescope for Astronomy (VISTA) as part of the VISTA near-infrared $Y, J, K_{\mathrm{s}}$ survey
\end{abstract}

\footnotetext{
${ }^{1}$ Kavli Institute for Astronomy \& Astrophysics, Peking University, Yi He Yuan Lu 5, Hai Dian District, Beijing 100871, China; joshuali@pku.edu.cn, grijs@pku.edu.cn

${ }^{2}$ Department of Astronomy, Peking University, Yi He Yuan Lu 5, Hai Dian District, Beijing 100871, China

${ }^{3}$ Key Laboratory for Optical Astronomy, National Astronomical Observatories, Chinese Academy of Sciences, 20A Datun Road, Chaoyang District, Beijing 100012, China

${ }^{4}$ INAF-Osservatorio Astronomico di Padova, vicolo dell'Osservatorio 5, I-35122 Padova, Italy

${ }^{5}$ ICRAR M468, The University of Western Australia, 35 Stirling Highway, Crawley, WA, 6009, Australia

${ }^{6}$ Department of Physics, Astronomy, and Mathematics, University of Hertfordshire, Hatfield AL10 9AB, UK

${ }^{7}$ Leibnitz-Institut für Astrophysik Potsdam, An der Sternwarte 16, D-14482 Potsdam, Germany

${ }^{8}$ INAF-Osservatorio Astronomico di Bologna, Via Ranzani 1, I-40127 Bologna, Italy

${ }^{9}$ Astronomy Unit, School of Physics and Astronomy, Queen Mary University of London, Mile End Road, London E1 4NS, UK

${ }^{10}$ Royal Observatory of Belgium, Ringlaan 3, 1180, Ukkel, Belgium

${ }^{11}$ Instituut voor Sterrenkunde, KU Leuven, Celestijnenlaan 200D 2401, 3001, Leuven, Belgium

${ }^{12}$ INAF-Osservatorio Astronomico di Capodimonte, via Moiariello 16, 80131 Naples, Italy

${ }^{13}$ Observatorio Astrońomico, Universidad Nacional de Córdoba, Laprida 854, 5000, Córdoba, Argentina

${ }^{14}$ Consejo Nacional de Investigaciones Científicas y Técnicas, Av. Rivadavia 1917, C1033AAJ, Buenos Aires, Argentina

${ }^{15}$ Astrophysics Group, Lennard-Jones Laboratories, Keele University, ST5 5BG, UK
} 
of the Magellanic System (VMC). The cluster stars comprising both the subgiant and red-giant branches exhibit apparent, continuous variations in colormagnitude space as a function of radius. Subgiant-branch stars at larger radii are systematically brighter than their counterparts closer to the cluster core; similarly, red-giant-branch stars in the cluster's periphery are bluer than their more centrally located cousins. The observations can very well be described by adopting an age spread of $\sim 0.5$ Gyr as well as radial gradients in both the cluster's helium abundance $(\mathrm{Y})$ and metallicity $(Z)$, which change gradually from $(\mathrm{Y}=0.28, Z=0.005)$ in the cluster core to $(\mathrm{Y}=0.25, Z=0.003)$ in its periphery. We conclude that the cluster's inner regions host a significant fraction of second-generation stars, which decreases with increasing radius; the stellar population in the 47 Tuc periphery is well approximated by a simple stellar population.

Subject headings: Hertzsprung-Russell and C-M diagrams — stars: abundances

— stars: sub-giant-branch — stars: red-giant-branch — stars: Population II

\section{Introduction}

Stars in a given star cluster are usually assumed to have originated from the same progenitor molecular cloud, from which they formed at approximately the same time. In turn, this implies that they would share the same metallicity (at least within a narrow range determined by the metallicity of the progenitor molecular cloud). These assumptions, which form the basis of the so-called (simple) single stellar population (SSP) model, have led to numerous very successful star cluster analyses. If we also assume that most stars evolve in isolation, then one can use a unique theoretical isochrone to describe the combination of main-sequence (MS), subgiant-branch (SGB), and red-giant-branch (RGB) stars. However, for many intermediate-age star clusters, and certainly for old globular clusters (GCs), the SSP approximation seems to break down.

The discovery of striking, extended MS turnoffs (eMSTOs) in many intermediate-age and old star clusters has led to a resurgence of the field of stellar population synthesis. Most authors suggest that the observed eMSTOs in, e.g., NGC 1783 (Mackey et al. 2008; Rubele et al. 2013), NGC 1846 (Mackey \& Broby Nielsen 2007; Rubele et al. 2013), or NGC 1868 (Li et al. 2014), can be adequately described by assuming a cluster-internal age dispersion of roughly 300 Myr (Rubele et al. 2010; Goudfrooij et al. 2011; Mackey et al. 2013), which hence challenges the applicability of the SSP approach. Alternative models, which maintain the SSP assumption, suggest that fast stellar rotation may be the cause of the observed 
eMSTOs (Bastian \& de Mink 2009; Bastian et al. 2013; Li et al. 2012, 2014; Yang et al. 2013) (but see Girardi et al. 2011; Platais et al. 2012). For some GCs, the breakdown of the SSP model assumptions is particularly convincing. For instance, some old GCs display double or multiple MSs, such as NGC 2808 (Piotto et al. 2007) and NGC 6397 (Milone et al. 2012a); in addition, Milone et al. (2008) found that NGC 1851 is characterized by two distinct SGBs. Some GCs - such as $\omega$ Centauri (Piotto et al. 2005; Sollima et al. 2007), NGC 288 (Piotto et al. 2013), and M22 (Lee et al. 2009) — exhibit double or multiple MSs, SGBs, and/or RGBs; Terzan 5 even shows clear, double HB clumps (Ferraro et al. 2009). All of these observations strongly indicate that at least some of the very old GCs contain multiple stellar populations.

The Galactic GC 47 Tucanae (47 Tuc; NGC 104) is another typical target cluster that convincingly displays multiple stellar population features across its entire color-magnitude diagram (CMD). It has also been known for a long time that 47 Tuc displays a clear nitrogen (N) dichotomy (Norris \& Freeman 1979, 1982; Norris et al. 1984). The cluster has been found to host at least two distinct MSs (Milone et al. 2012b), a broadening of the SGB or (alternatively) multiple SGBs (Anderson et al. 2009; Milone et al. 2012b), as well as double or multiple RGBs (Milone et al. 2012b; Monelli et al. 2013). In addition, Nataf et al. (2011) analyzed the radial gradients in RGB-bump and horizontal-branch (HB) stars, which indicate the presence of a helium-enriched second stellar generation in 47 Tuc. Cordero et al. (2014) determined a $\mathrm{Na}-\mathrm{O}$ anticorrelation in $47 \mathrm{Tuc}$, a signature typically seen in Galactic GCs (e.g., Carretta et al. 2009) and which indicates contamination by products of the proton-capture process (Langer et al. 1993). In other GCs (e.g., in M71; see Ramírez \& Cohen 2002) such anticorrelations are even found in some less evolved MS stars, which have not reached sufficiently high temperatures to trigger proton capture. This implies the presence of more than one stellar population in Galactic GCs.

Different origins have been proposed to account for the secondary stellar generations; they mainly invoke scenarios involving accretion of the ejecta of intermediate-mass stars (D'Antona et al. 1983; Renzini 1983), rapidly rotating massive stars (Decressin et al. 2007), massive binaries (de Mink et al. 2009), and evolved asymptotic-giant-branch stars (Ventura \& D'Antona 2009). Previous studies have shown that second-generation stars originating from their first-generation counterparts are generally more centrally concentrated (Decressin et al. 2008; D'Ercole et al. 2008; Bekki 2011). ${ }^{1}$ Indeed, in 47 Tuc Milone et al. (2012b)

\footnotetext{
${ }^{1}$ Full mixing of both populations will take at least 20 half-mass relaxation times (cf. Vesperini et al. 2013). Note that the half-mass radius of 47 Tuc is $174^{\prime \prime}$ (Trager et al. 1993), which implies that the vast majority of the cluster's member stars analyzed here are located outside of the cluster's half-mass radius and thus unrelaxed.
} 
investigated the radial behavior of two distinct RGB populations, as well as that of two subgroups of HB stars. They concluded that second-generation stars gradually start to dominate at increasingly smaller radii. This conclusion was confirmed by Cordero et al. (2014, their figure 4) based on chemical abundance analysis. Anderson et al. (2009) explored the global characteristics of the SGB stars in 47 Tuc. They pointed out that the broadening of the SGB in the 47 Tuc CMD indicates the presence of more than one stellar population, but because of constraints inherent to their sample selection, they could not investigate a promising radial trend related to a possible second generation of SGB stars in 47 Tuc.

In this article, we present a deep, large-area, near-infrared (NIR) CMD of 47 Tuc, obtained with the $4 \mathrm{~m}$ Visible and Infrared Survey Telescope for Astronomy (VISTA). We confirm that the cluster's SGB and RGB are significantly broadened, which cannot be explained as simply owing to the effects of either photometric uncertainties or (differential) extinction. Compared with Milone et al. (2012b), the RGB's radial behavior we find displays a more obvious radial color bias. We find that the average color of the RGB stars at large radii is significantly different from that in the innermost regions. The apparent color gradient strongly indicates that the two RGB populations contain significantly different fractions of stars formed as part of a second stellar generation. Following Anderson et al. (2009), we also find that the cluster's SGB is significantly broadened. The SGB stars in the periphery are on average significantly brighter than the innermost SGB stars. All of these features are so striking that they can even be clearly seen based on a single, quick glance at the cluster's global CMD. The present paper focuses on new, high-quality NIR observations of 47 Tuc, which offers an excellent comparison data set to the ultraviolet/optical study of Milone et al. (2012b). We also highlight that our observations cover a significantly larger field than that of Milone et al. (2012b): our data set's maximum distance to the cluster's central region reaches $4000^{\prime \prime}$ (compared with the radial range out to $1500^{\prime \prime}$ available in the earlier publication). ${ }^{2}$ We can thus very well characterize the effects caused by background contamination.

This article is organized as follows. In Section 2 we describe the data reduction approach. Section 3 presents the main results, which we discuss in Section 4. A brief summary and our conclusions are presented in Section 5.

\footnotetext{
${ }^{2}$ At the cluster's distance of $4.6 \mathrm{kpc}$ (derived below), $1^{\prime \prime} \equiv 0.022 \mathrm{pc}$.
} 


\section{Data Reduction and Analysis}

The data set analyzed in this article was obtained as part of the VISTA near-infrared $Y, J, K_{\mathrm{s}}$ survey of the Magellanic System (VMC; ${ }^{3}$ PI M.-R. L. Cioni: see Cioni et al. 2011). The survey started in November 2009 and is expected to extend beyond the originally planned 5 yr time span (survey completion is foreseen for 2018; Arnaboldi et al. 2013). Its main goals and first data are described in Cioni et al. (2011). 47 Tuc is fortuitously located in front of the Small Magellanic Cloud (SMC), on VMC tile "SMC 5_2," which made it an ideal target for early VMC data acquisition.

The VMC images were reduced by the Cambridge Astronomy Survey Unit (CASU) using the VISTA Data Flow System (VDFS) pipeline (Irwin et al. 2004). To perform our pointspread-function (PSF) photometry starting from the pawprint VMC images, we selected - in all three filters $\left(Y, J, K_{\mathrm{s}}\right)$ - epochs with limited seeing inhomogeneities. The selected reduced data, comprising two epochs in $Y$ and $J$ and nine in $K_{\mathrm{s}}$, are PSF-homogenized and stacked to obtain a deep tile image (using a novel method that will be described in detail by Rubele et al., in prep.). We performed PSF photometry on the deep image of tile SMC 5_2, using the IRAF DAOPHOT package (Stetson 1987). The PSF and ALLSTAR tasks were used to produce PSF models and perform the final photometry. We checked and corrected our PSF photometry for aperture effects using catalogs retrieved from the VISTA Science Archive (Cross et al. 2012) for the bulk of the observed stars.

Here, we focus our analysis on the $\left(Y, Y-K_{\mathrm{s}}\right) \mathrm{CMD}$, where the features of interest are most apparent, because this color extends over the longest available wavelength range. However, we confirmed that our conclusions also hold based on analysis of the $(Y, Y-J)$ and $\left(J, J-K_{\mathrm{s}}\right)$ CMDs. Our observations cover an area of roughly $1.2 \times 5 \mathrm{deg}^{2}$. The bulk of the 47 Tuc stars are located toward the southwest of this region, so that the opposite corner of the field is suitable for analysis of the field-star properties, which we will use to decontaminate the cluster CMD.

47 Tuc is an extremely large and crowded GC. In our data, it covers more than 300,000 stars with $Y \in[11.0,25.5]$ mag. Because of the effects of mass segregation (cf. de Grijs et al. 2002a,b,c), almost all of the more massive $\left(m_{*} \geq 0.88 M_{\odot}\right)$, bright $(Y \leq 13 \mathrm{mag})$ cluster stars are contained within the central region, which dramatically increases the background level in the cluster core because of the extended, faint wings of the PSF profile. In the central region, almost all stars fainter than $Y \sim 13-15$ mag will fade into the background because of a combination of the enhanced background level and stellar crowding in our ground-based

\footnotetext{
${ }^{3}$ http://star.herts.ac.uk/ mcioni/vmc
} 
images. For these reasons, we only use the 3071 stars brighter than $Y=13.0$ mag, in essence HB stars and a fraction of the cluster's bright RGB and asymptotic-giant-branch stars, to determine the cluster center. Our approach to find the cluster center is identical to that employed by de Grijs et al. (2013) and Li et al. (2013, 2014).

We first selected a sufficiently large rectangular area that clearly contained the full cluster region and then divided the stellar spatial distributions into 500 bins in both the right ascension $\left(\alpha_{\mathrm{J} 2000}\right)$ and declination $\left(\delta_{\mathrm{J} 2000}\right)$ directions. In both spatial coordinates, the stellar number densities follow Gaussian-like profiles, which allow us to determine the coordinates corresponding to the two-dimensional (2D) maximum stellar density. The resulting center coordinates are $\alpha_{\mathrm{J} 2000}=00^{\mathrm{h}} 24^{\mathrm{m}} 04.80^{\mathrm{s}}\left(6.020^{\circ}\right.$; statistical uncertainty $\left.0.007^{\circ} \equiv 25^{\prime \prime}\right), \delta_{\mathrm{J} 2000}=$ $-72^{\circ} 04^{\prime} 48^{\prime \prime}\left(-72.080^{\circ} \pm 0.006^{\circ} \equiv 20^{\prime}\right)$. Our approach introduces negligible biases, because the member stars of extremely old GCs should all have adapted their kinematics to be in equilibrium with the prevailing gravitational potential, so that both the bright stars (which we excluded from our analysis) and their fainter companions experience the same gravitational potential. Our result is consistent with previous determinations of the cluster center by McLaughlin et al. (2006), Goldsbury et al. (2010), and Harris (2010).

We subsequently selected a second region centered at $\alpha_{\mathrm{J} 2000}=00^{\mathrm{h}} 31^{\mathrm{m}} 59.00^{\mathrm{s}}\left(8.00^{\circ}\right)$, $\delta_{\mathrm{J} 2000}=-71^{\circ} 42^{\prime} 00^{\prime \prime}\left(-71.70^{\circ}\right)$, with a radius of $600^{\prime \prime}$, to calculate the background stellar number density (for stars with $Y \leq 13.0 \mathrm{mag}$ ). The vast majority of stars in this region are foreground Milky Way and background SMC stars; contamination by 47 Tuc members at these large radii is $\ll 15 \%$, an estimate based on careful assessment of the cluster's extended radial profile. This region is a suitable choice for a "field" region, because its center is located more than $2400^{\prime \prime}$ from the cluster center, a distance that is close to the cluster's tidal radius $\left(r_{\mathrm{t}}=56 \mathrm{pc} \equiv 2500^{\prime \prime}\right.$ for a distance modulus of $(m-M)_{0}=13.40 \mathrm{mag}$ or a distance of 4.6 kpc, derived below; see Harris 1996; Lane et al. 2012); this is unlikely to significantly affect our field-star characterization. We cannot rule out the presence of a significant population of extratidal stars, however. Lane et al. (2012) predicted that 47 Tuc should exhibit at least one extratidal tail, which would cross our observational field of view from the northwest to the southeast. We hence selected a region in the northeast of our field of view as field reference so as to avoid contamination by extratidal-tail stars as much as possible. In fact, we found that the stellar number density in the southeast is indeed significantly higher than that in the northeast. We next compared the cluster's stellar number density with that of the background field to check at which radius, $R_{\mathrm{f}}$, the cluster's stellar number density becomes indistinguishable from the background level. We determined $R_{\mathrm{f}}=1220 \pm 20^{\prime}$, and hence adopted a cluster "size" of $1200^{\prime \prime}$ for further study, equivalent to approximately $50 \%$ of its tidal radius. This is a conservative choice, because we eliminated the brightest stars from our analysis. Mass segregation will cause an underestimation of 
the "bright" cluster size compared with that resulting from having adopted a homogeneous stellar sample. However, even if we were to select a smaller cluster size, our results still strongly indicate the presence of multiple stellar generations.

Figure 1 displays the stellar spatial distribution of stars brighter than $Y=17$ mag. We also show the stars in the adopted field region (blue circle), characterized by a radius of $600^{\prime \prime}$. The cluster region is indicated by the red circle. In Figure 2 we display the radial stellar number-density profile (on a logarithmic scale), where the black solid line indicates the field stellar density level and the vertical black dashed line indicates the radius, $R_{\mathrm{f}}$, where the cluster's stellar number density becomes indistinguishable from that of the background.

Next, we adopted the most suitable isochrone describing our photometric data, using model isochrones based on the PGPUC stellar evolution code ${ }^{4}$ (Valcarce et al. 2012, with $Z_{\odot}=0.0152$ as its basis). We adopted an age for 47 Tuc of 12.5 Gyr or $\log \left(t \mathrm{yr}^{-1}\right)=10.10$ (cf. Zoccali et al. 2001; McLaughlin et al. 2006; Di Criscienzo et al. 2010; McDonald et al. 2011) and a metallicity of $Z=0.0042$ or $[\mathrm{Fe} / \mathrm{H}]=-0.55 \mathrm{dex}$. The latter value is consistent with the determination of Nataf et al. (2011) (Note that these authors adopted $[\mathrm{M} / \mathrm{H}]=-0.52$ dex instead of $[\mathrm{Fe} / \mathrm{H}] ;[\mathrm{M} / \mathrm{H}]=A \times[\mathrm{Fe} / \mathrm{H}]$, where $A \in[0.9,1.0]$. We simply adopt an average value of $A=0.95$.) The adopted extinction, $E(B-V)=0.04 \mathrm{mag}$, is equal to that determined and adopted by Harris (1996), Anderson et al. (2009), and Cordero et al. (2014). We also need to adopt a suitable value for the $[\alpha / \mathrm{Fe}]$ ratio. Carretta et al. (2009) determined $[\alpha / \mathrm{Fe}]=0.40$ dex. We hence adopted the maximum value available in the PGPUC code, $[\alpha / \mathrm{Fe}]=0.30 \mathrm{dex}$, to generate the most representative isochrone for the bulk of the cluster stars; we found that this small offset between the most appropriate and closestavailable model- $[\alpha / \mathrm{Fe}]$ values will not introduce significant fitting problems. Based on these input parameters, the best-fitting distance modulus to 47 Tuc is $(m-M)_{0}=13.40 \pm 0.10$ mag, which is consistent with the determinations of Harris (1996), Zoccali et al. (2001), and McDonald et al. (2011).

We next performed field-star decontamination. As illustrated in Figure 1, we selected stars from a suitably chosen nearby region as our reference to statistically correct for background contamination. We selected a field region covering a circular area with a radius of 600 ", which corresponds to one quarter of the adopted cluster area, and compared the corresponding CMDs. For every star in the CMD of the field region, we removed the closest four counterparts (thus correcting for the difference in the cluster versus field areas adopted) in the corresponding CMD of the cluster. Although any such star may not individually be a genuine field star, this approach nevertheless ensures that the cluster CMD is decontami-

\footnotetext{
${ }^{4}$ http://www2.astro.puc.cl/pgpuc/iso.php
} 




Fig. 1.- Spatial distribution of 47 Tuc stars with $Y \leq 17.0$ mag. North is up; East is to the left. The red pentagram represents the adopted cluster center, the red circle indicates the cluster region of interest, corresponding to a radius of $1200^{\prime \prime}$, and the blue circle maps out a representative region adopted for field-star decontamination, with a radius of $600^{\prime \prime}$. The small group of stars located at approximately $\left(\alpha_{\mathrm{J} 2000} \sim 6.7^{\circ}, \delta_{\mathrm{J} 2000} \sim-71.5^{\circ}\right)$ is the $\mathrm{SMC}$ cluster NGC 121 . The center of the SMC is located at approximately $1.7^{\circ}$ toward the southeast of 47 Tuc.

nated statistically robustly (e.g., de Grijs et al. 2013; Li et al. 2013, 2014). In addition, we mainly focus on SGB and RGB stars, while very few of the field stars in the region adopted for field-star decontamination are this bright. This hence supports the reliability of our statistical decontamination approach.

Figure 3 shows the decontaminated cluster CMD, including all stars located within $1200^{\prime \prime}$ of the cluster center, as well as the most appropriate isochrone given the cluster's 


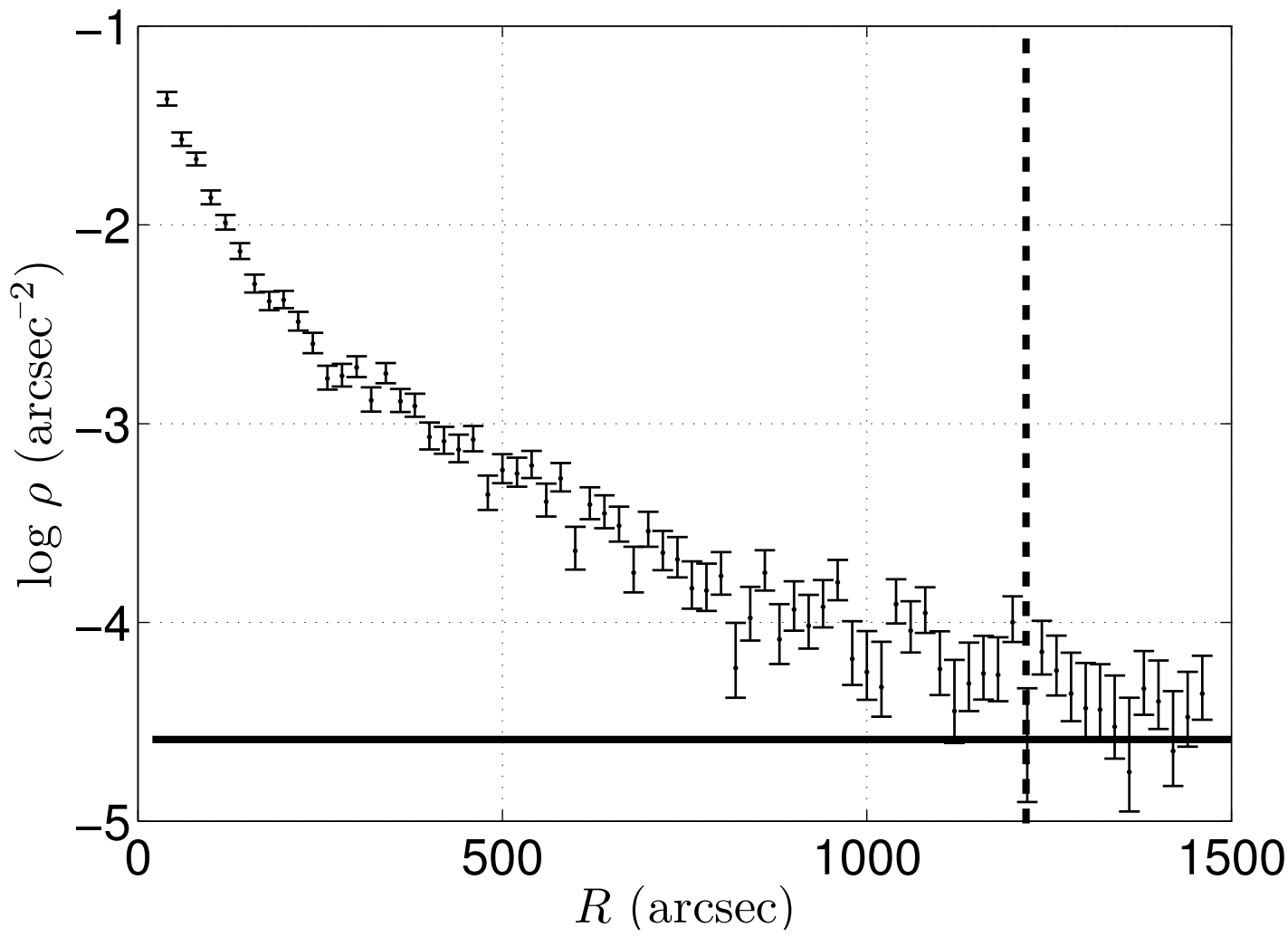

Fig. 2.- Logarithmic radial number-density profile of stars brighter than $Y=13$ mag. The horizontal solid line indicates the background level, while the vertical dashed line signifies the adopted cluster size.

overall parameters. The color scale represents the distance to the cluster center. A quick glance at this figure already suggests a systematic difference between the cluster stars in the innermost region and in the cluster's periphery. Even if we ignore the MS stars due to a lack of accurate data in the cluster's core region (because of low completeness at these relatively faint magnitudes in the inner region; see Figure 4), the SGB and RGB stars exhibit a clear difference as a function of radius. SGB stars at large radii are systematically brighter than those located in the central regions; they join the RGB at clearly bluer colors compared with the color range characteristic of the innermost RGB stars, hence implying higher characteristic temperatures. All stars shown in Figure 3 have photometric uncertainties of less than 0.02 mag. It is thus clear that the broadening of both the SGB and the RGB cannot be only owing to photometric uncertainties.

Because of the complicated physical conditions affecting our 47 Tuc observations (including those caused by significant mass segregation and frequent stellar blending due to 


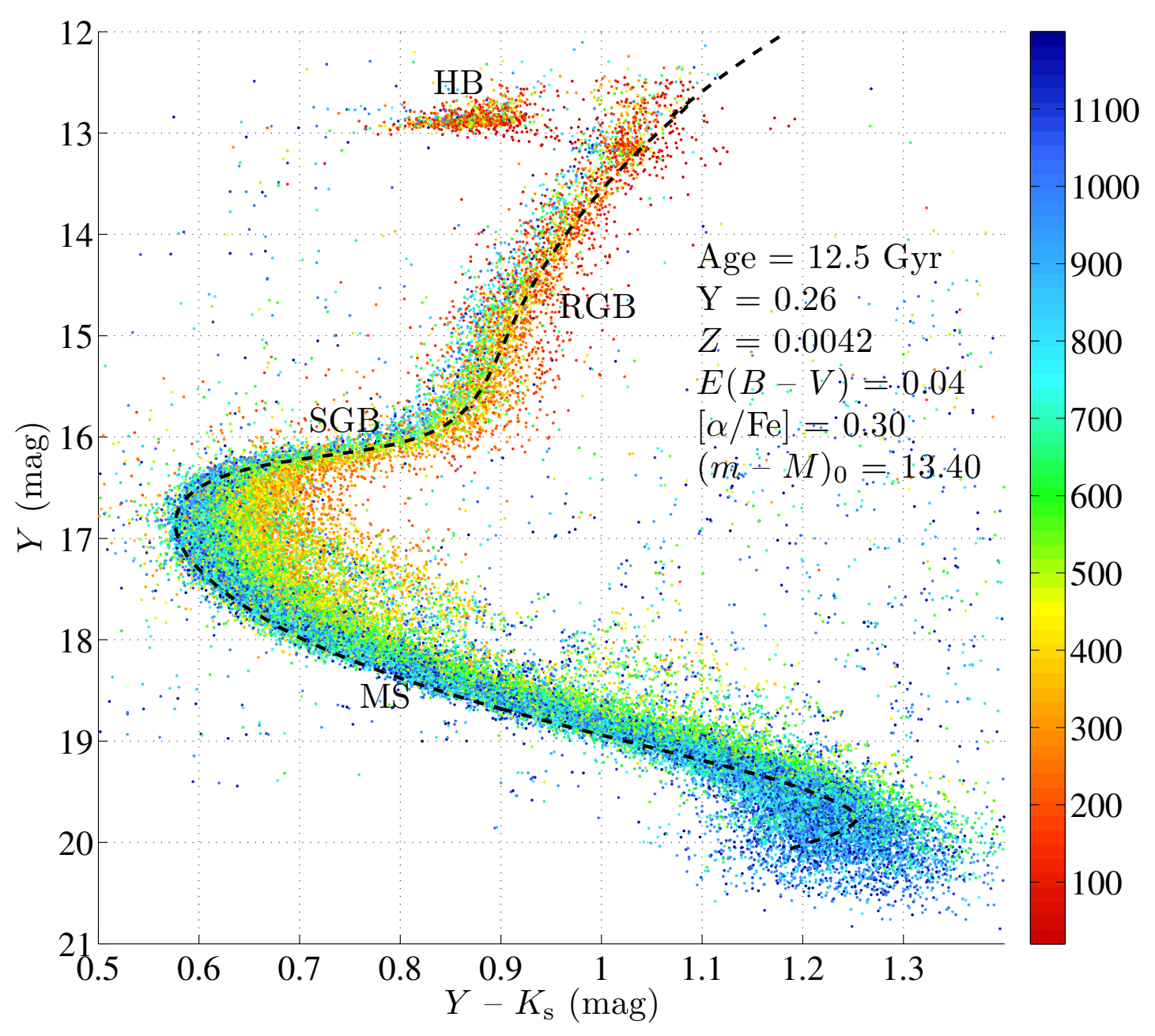

Fig. 3.- $\left(Y, Y-K_{\mathrm{s}}\right) \mathrm{CMD}$ of 47 Tuc, showing only stars with photometric uncertainties of $\leq 0.02 \mathrm{mag}$ in all filters. The color scale indicates the distance to the cluster center (in arcsec). The black dashed line represents the best-fitting isochrone.

crowding effects), most faint stars have very large photometric uncertainties, while some of the brightest stars $\left(K_{\mathrm{s}} \lesssim 11.5 \mathrm{mag}\right)$ are partially saturated. This latter effect also introduces large photometric uncertainties. We therefore removed those stars in our overall sample that have photometric uncertainties in excess of $0.02 \mathrm{mag}$ in all three filters. This resulted in a final working sample that contained the $\sim 30 \%$ of stars with the highest-accuracy photometry in the corresponding magnitude range. Note that because of the crowding in the cluster's core region, more centrally located stars will have larger errors (for the same source 
brightness), which hence may introduce an additional bias. To quantify such a bias, we used artificial-star (AS) tests to study the systematic effects of crowding on the uncertainties in the resulting PSF photometry. We added $7.2 \times 10^{6}$ ASs to the raw image and subsequently measured them in the same manner as our sample of real stars, using PSF photometry. The number of ASs is roughly 20 times larger than the number of real stars. To avoid a situation in which the ASs affect or even dominate the background level and cause crowding artifacts, we only add 3500 stars to the raw image at any given time. Although this choice implies that there is a (very small) probability that two ASs may blend with each other, adopting a much smaller number of ASs at a time would significantly increase the computational cost of our AS tests. (We quantified the effects of ASs blending with other ASs, and found the possibility of such a situation occurring to be negligible.) We repeated this procedure 2000 times. The distribution of the ASs followed a spatial distribution that reproduced the cluster shape derived from bright stars, as well as a distribution in the color-magnitude diagram that resembled the real distribution. The resulting AS catalog contains the input and recovered (output) magnitudes, as well as the photometric errors computed as output - input magnitudes.

We carefully assessed any systematic effects of the photometric uncertainties on our PSF photometry owing to crowding. The main result of these AS tests is that the positions in the CMD of the SGB and RGB stars that we are concerned with in this study are negligibly affected. However, the photometric errors for stars in the central region are larger than the errors resulting from the use of DAOPHOT, for the same object, while there is good agreement between both sets of photometric-error measurements in the outer regions, as expected. This effect only becomes important for stars that are fainter than the MSTO; for their brighter counterparts the deviation is negligible. Our results hence confirm that the spread in the SGB/RGB is real.

The AS tests also allow us to estimate the photometric completeness of our stellar catalog. The crowding in the cluster's central regions affects the ASs in a similar way as the real cluster members. We generated artificial stars that were homogeneously distributed across the same region as the observations. Those ASs that returned either no photometric measurement or that were characterized by a difference in output-input magnitude that was greater than five times their photometric uncertainties were considered stars that could not be recovered (hence tracing the level of completeness of the observations). Figure 4 displays the cumulative 2D completeness map as a function of both radius and $Y$ magnitude. 


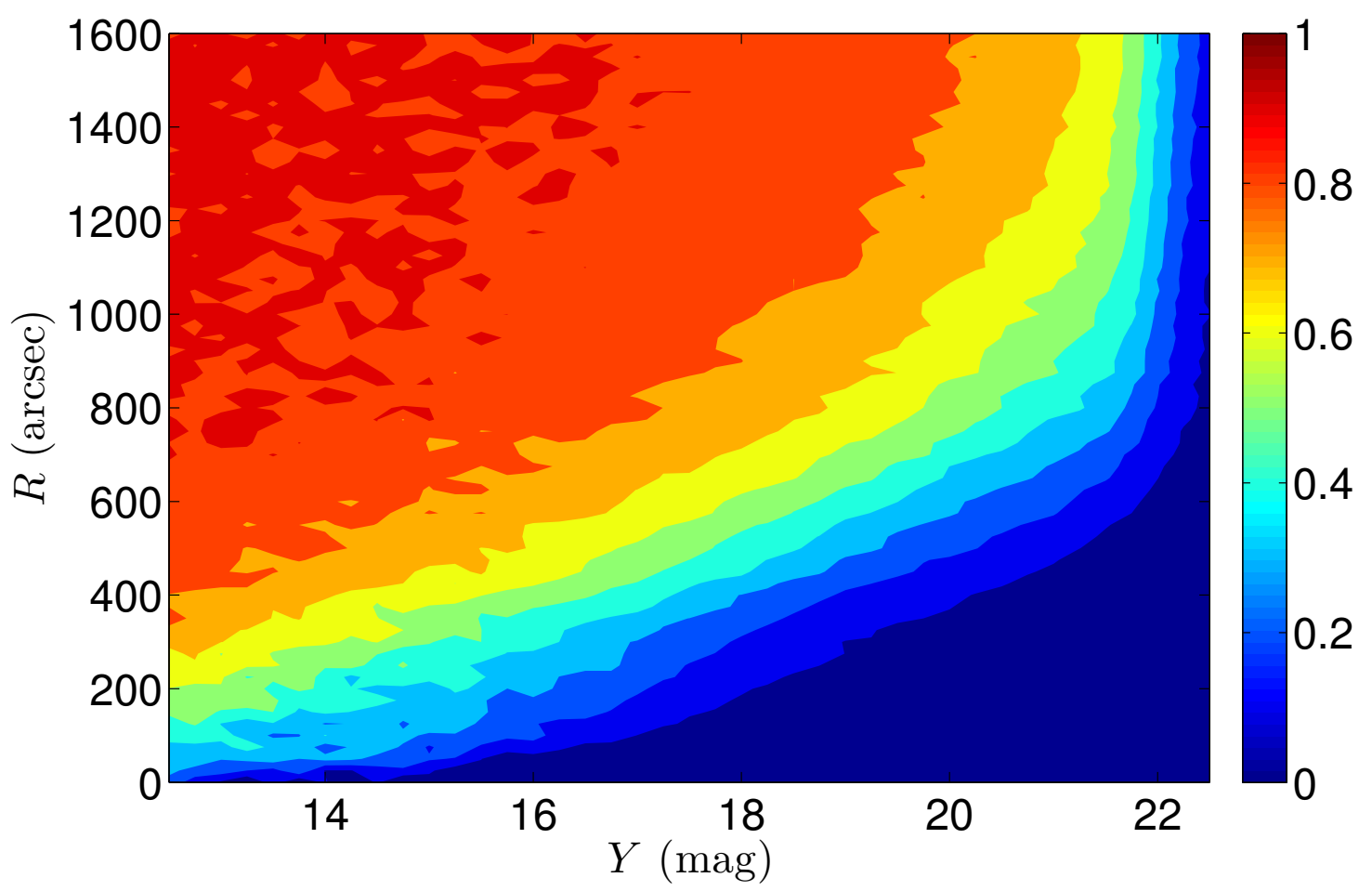

Fig. 4. - 2D completeness map as a function of radius and $Y$ magnitude.

\section{Results}

\subsection{Subgiant-branch stars}

We start by focusing on the region occupied by the SGB stars. First, we select a box delineated by $Y \in[15.5,16.7] \mathrm{mag}$ and $\left(Y-K_{\mathrm{s}}\right) \in[0.70,0.85] \mathrm{mag}$. Although this selection is quite arbitrary, we only need to ensure that the region contains the vast majority of SGB stars. Because the photometry of stars in this magnitude range is more accurate than that of the faint(er) MS stars, we further impose a maximum photometric uncertainty of 0.015 mag. This constraint leaves us with the $\sim 30 \%$ of stars with the highest-accuracy photometry in the corresponding part of CMD space.

We next adopt the cluster-wide ridge line as our standard model. The locus of the SGB ridge line is determined by connecting the loci of the maximum stellar number density as a function of color. We calculate the magnitude difference with respect to this observational ridge line for all stars in our SGB sample, $\Delta Y=Y_{\mathrm{SGB}}-Y_{\text {iso }}$. The resulting magnitude dispersion is distributed in a Gaussian-like fashion with $\sigma_{\Delta Y} \sim 0.18$ mag. We remove stars that were found beyond $3 \sigma_{\Delta Y}=0.54 \mathrm{mag}$ of the ridge line, resulting in a sample of 1389 
stars: see Figure 5.

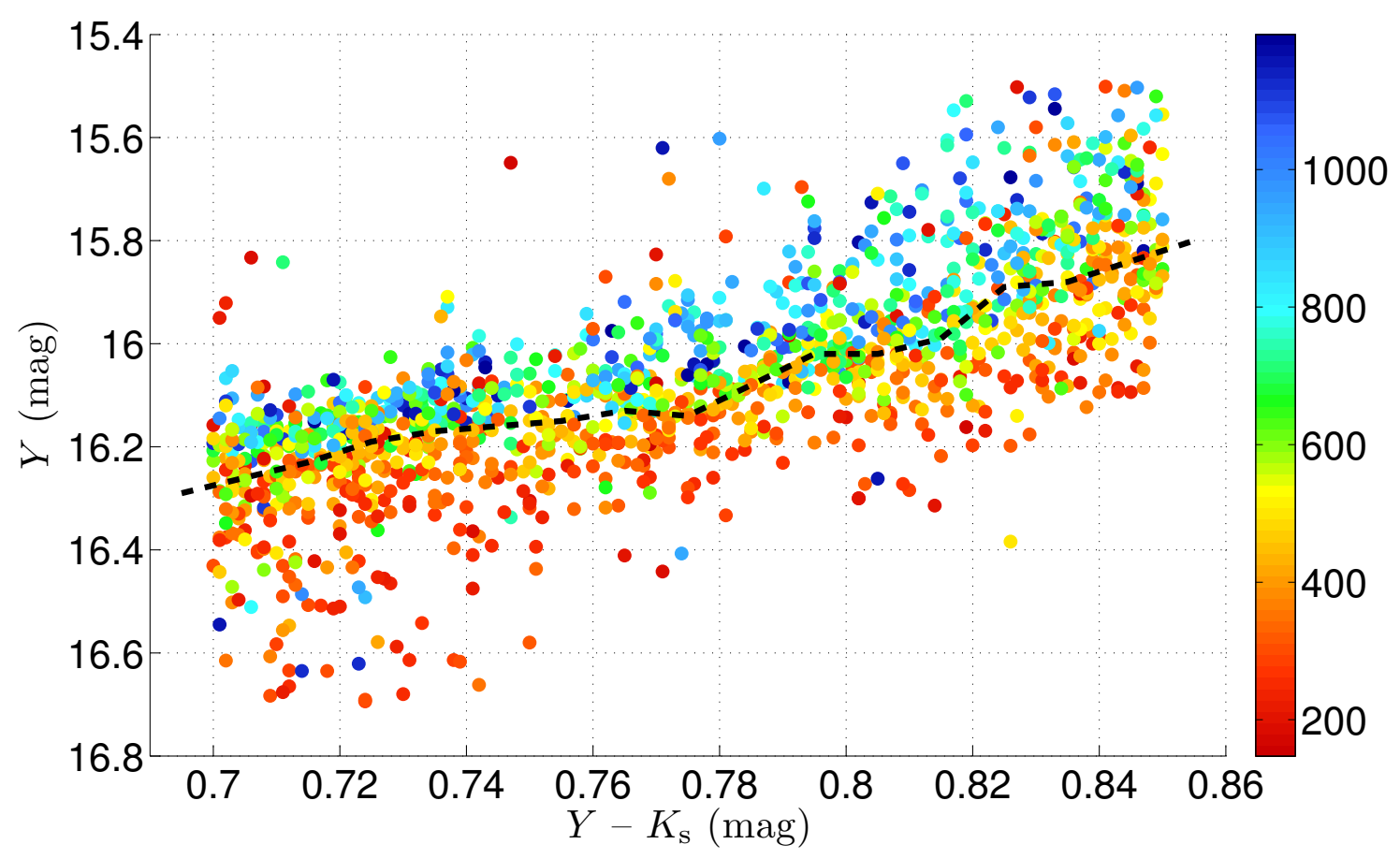

Fig. 5.- CMD of the adopted SGB region. The color scale indicates the distance to the cluster center (in arcsec). Because of sampling incompleteness in the cluster core, the minimum radius attainable is $\sim 150^{\prime \prime}$. All stars have magnitudes within $3 \sigma_{\Delta Y}=0.54 \mathrm{mag}$ of the SGB ridge line.

Figure 5 shows that the SGB stars in the cluster's periphery are systematically found above the ridge line, i.e., they are brighter compared with their more centrally located counterparts. We divide the SGB sample into five annuli as a function of radius to check whether and - if so - how their magnitude dispersion varies with radius. The four boundaries between subsequent annuli were set at $340^{\prime \prime}, 450^{\prime \prime}, 590^{\prime \prime}$, and $800^{\prime \prime}$, which resulted in roughly equal stellar numbers (ranging from 269 to 283) per radial bin. For each radial bin, we calculate the distribution of the stellar magnitude dispersion, normalized to the total number of stars in the relevant bin. We use these distributions to calculate the probability distribution of $\Delta Y$ in each radial bin, as shown in Figure 6.

Based on Figure 6, it is clear that the SGB stars in the innermost radial bin (top panel) are systematically fainter $(\Delta Y>0 \mathrm{mag})$ than those in the cluster's periphery (bottom panel). As a function of increasing radius, the mean magnitude of the bulk of the SGB stars becomes gradually brighter $(\Delta Y<0 \mathrm{mag})$. We also note that for the central sample, the 

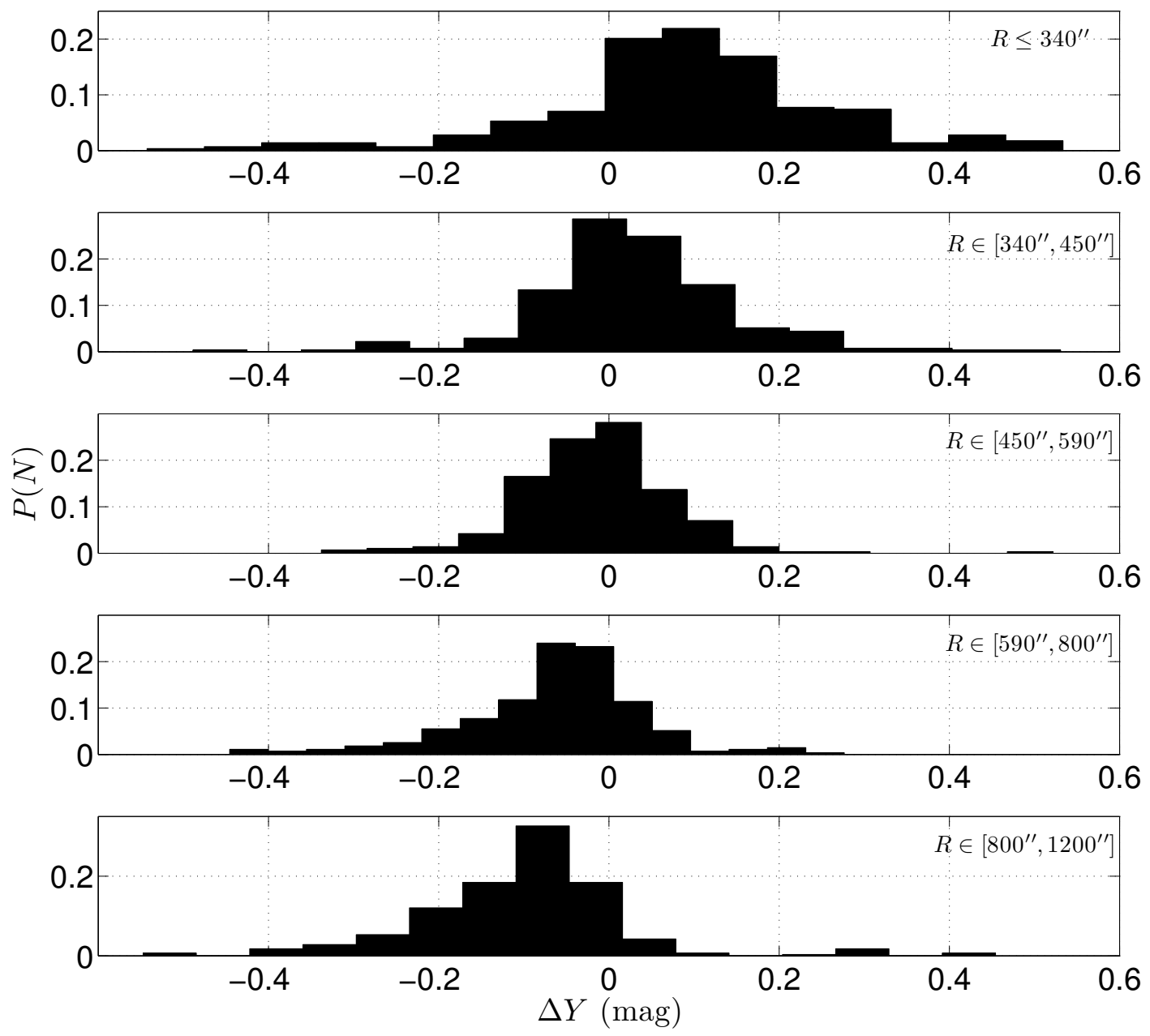

Fig. 6. - Probability distribution of $\Delta Y$ as a function of radius for SGB stars in 47 Tuc. From top to bottom, the panels represent radial bins from the cluster's inner regions to its outskirts.

magnitude dispersion of the central SGB stars is larger than that of the peripheral SGB sample. This is as expected, because the observed stellar distribution is a $2 \mathrm{D}$ projection onto the plane of the sky: the stars in the peripheral sample are indeed located far from the cluster center, but the innermost stars are significantly contaminated by stars that are physically located at larger radii. Therefore, if the properties of the SGB stars at large radii differ systematically from those in the innermost sample, the contaminated innermost sample will exhibit a larger magnitude dispersion. 
In addition, blending of unresolved stars that are physically not associated with each other but located along the same line of sight will also cause a dispersion of SGB stars to brighter magnitudes. Such blending events can happen anywhere, but they are expected to occur more frequently in the cluster's innermost regions, where the stellar number density is highest. Both of these effects will cause a bias to brighter magnitudes that predominantly affects the innermost SGB stars. It is thus natural to expect that the intrinsic distribution of SGB stars in the cluster core would be narrower than observed. In Figure 7 we show the CMD of SGB stars at $R \leq 340^{\prime \prime}$ (i.e., the innermost radial bin), as well as that composed of SGB stars at $R \in\left[800^{\prime \prime}, 1200^{\prime \prime}\right]$ (i.e., the outermost radial bin adopted). It is clear that the most centrally located SGB stars are more dispersed (in magnitude) than the peripheral SGB stars; both samples are clearly different.

We use a Monte Carlo method to estimate how many projected stars at large radii will contaminate the stellar sample drawn from the inner regions (cf. Li et al. 2014). For example, if we want to estimate the contamination of SGB stars in the ring defined by $R \in\left[R_{1}, R_{2}\right]$, where $R_{2}>R_{1}$, we first calculate how many SGB stars are located at $R \geq R_{2}$, say $N=N\left(R \geq R_{2}\right)$. We then assume that these $N$ stars are located in a three-dimensional (3D) spherical shell at $R \in\left[R_{2}, R_{\mathrm{f}}\right]$, where $R_{\mathrm{f}}=1200^{\prime \prime}$ is the cluster size. The 3D stellar density at $R \in\left[R_{2}, R_{\mathrm{f}}\right]$ is then

$$
\rho=\frac{3 N}{4 \pi\left(R_{\mathrm{f}}^{3}-R_{2}^{3}\right)} .
$$

We next generate a 3D spherical cluster of size $R_{\mathrm{f}}$, which hence contains $N_{\text {art }}=\left(4 \pi R_{\mathrm{f}}^{3} / 3\right) \times \rho$ artificial stars. The artificial stars are homogeneously distributed with density $\rho$. We now randomly select a given direction to represent the line-of-sight direction; the number of stars located at $R \geq R_{2}$ that will be projected at $R_{\text {proj }} \in\left[R_{1}, R_{2}\right]$ is referred to as $N_{\text {proj. For }}$ each radial bin, we repeat this process 10 times to calculate the average $N_{\text {proj }}$ and obtain an approximate projected number of stars in the radial annulus of interest. Because only stars at $R \geq R_{2}$ will cause contamination of a ring at $R \in\left[R_{1}, R_{2}\right]$, even if we assume that the average density of stars at $R \geq R_{2}$ is homogeneous and that they are spherically distributed, which will underestimate the real stellar density in the region at $R \leq R_{2}$, this will not affect our estimation.

The resulting ratio of the projected contamination indicates, for example, that for the sample drawn from the (projected) innermost radii, $R \leq 340^{\prime \prime}, 103$ stars are actually physically located at $R>340^{\prime \prime}$. Similarly, for the radial range $R \in\left[590^{\prime \prime}, 800^{\prime \prime}\right]$, only 43 stars will be located beyond $R=800^{\prime \prime}$ along the line-of-sight direction.

If we normalize the total number of stars that we observe in each projected radial bin, the contamination due to line-of-sight projection decreases from $36 \%$ for the innermost radii 
$\left(R \leq 340^{\prime \prime}\right)$ to $16 \%$ for the penultimate radial range $\left(R \in\left[590^{\prime \prime}, 800^{\prime \prime}\right]\right)$. We have assumed that for radii $R \in\left[800^{\prime \prime}, 1200^{\prime \prime}\right]$ the effects of projection are negligible. Because more than half of the central SGB stars are, in fact, peripheral stars that have been projected along the line-of-sight, it is not strange that the magnitude dispersion of the innermost SGB stars is larger than that of the peripheral sample(s). Table 1 includes the details of the projected contamination fraction derived this way, for both SGB and RGB stars (for a discussion of the latter, see Section 3.2).

Table 1: Contamination due to line-of-sight projection for SGB and RGB stars as a function of radius.

\begin{tabular}{l|c||l|c}
\hline \hline SGB stars & $f_{\text {proj }}$ & RGB stars & $f_{\text {proj }}$ \\
\hline \hline$R \leq 340^{\prime \prime}$ & $36.40 \%$ & $R \leq 260^{\prime \prime}$ & $29.00 \%$ \\
$R \in\left[340^{\prime \prime}, 450^{\prime \prime}\right]$ & $20.82 \%$ & $R \in\left[260^{\prime \prime}, 370^{\prime \prime}\right]$ & $20.55 \%$ \\
$R \in\left[450^{\prime \prime}, 590^{\prime \prime}\right]$ & $20.42 \%$ & $R \in\left[370^{\prime \prime}, 510^{\prime \prime}\right]$ & $19.46 \%$ \\
$R \in\left[590^{\prime \prime}, 800^{\prime \prime}\right]$ & $15.87 \%$ & $R \in\left[510^{\prime \prime}, 730^{\prime \prime}\right]$ & $16.26 \%$ \\
\hline \hline
\end{tabular}

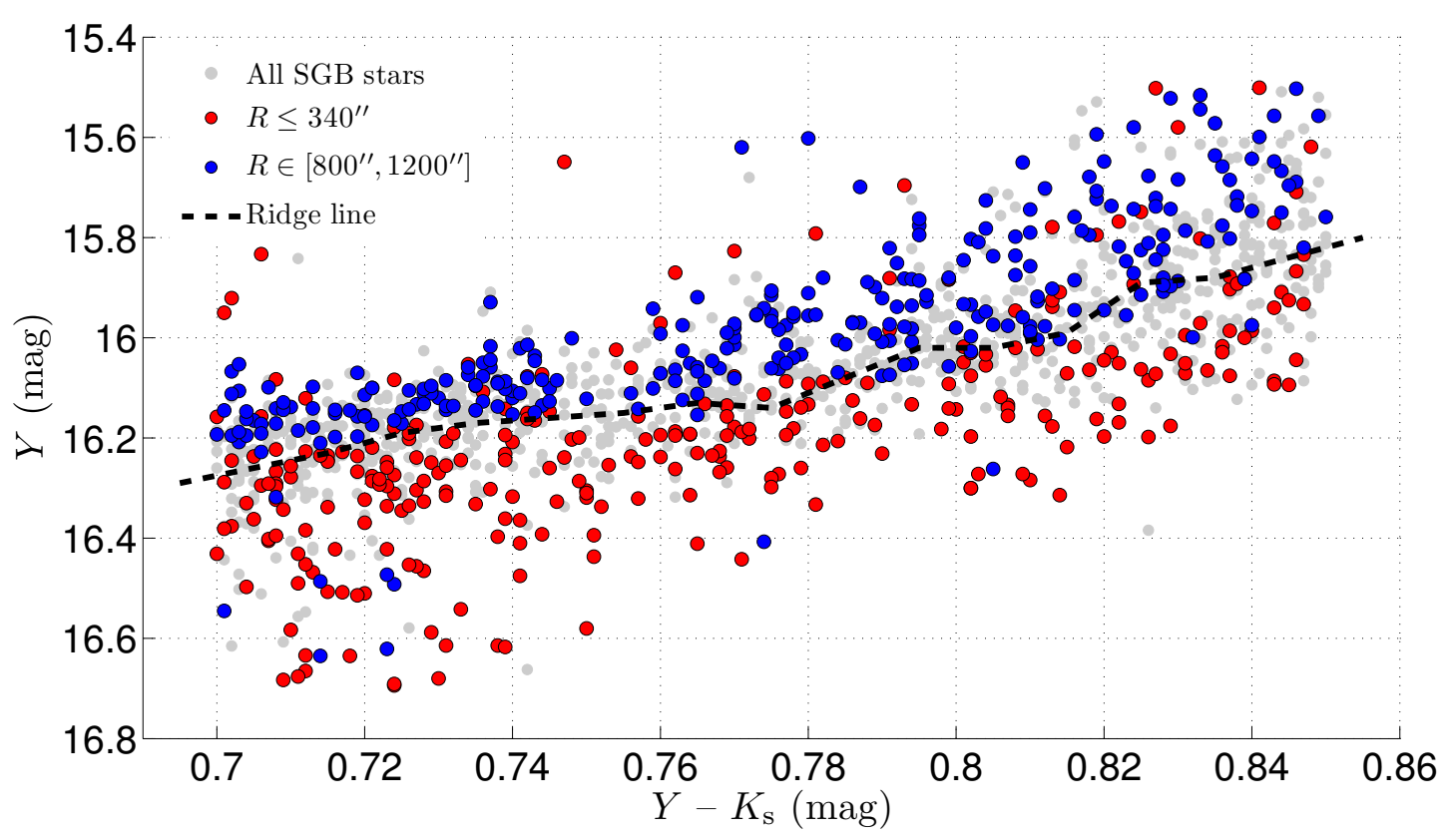

Fig. 7.- CMD of the most centrally located SGB stars in 47 Tuc $\left(R \leq 340^{\prime \prime}\right.$ : red solid bullets) and the outermost SGB stars $\left(R \in\left[800^{\prime \prime}, 1200^{\prime \prime}\right]\right.$ : blue solid bullets). Smaller grey bullets represent all SGB stars in our sample.

We show the 2D contours of the SGB stars' probability distribution, $P(\Delta Y, R)$, as a function of $\Delta Y$ and radius in Figure 8. This figure strongly indicates the presence of at 
least two SGB populations, the relative fractions of which clearly exhibit a gradual change in terms of their magnitudes. One population is mainly concentrated at radii between $R=600^{\prime \prime}$ and $R=900^{\prime \prime}$, while the second population peaks between $R=400^{\prime \prime}$ and $R=500^{\prime \prime}$. It is possible that a third peak is associated with the innermost cluster regions, but the significant magnitude dispersion due to contaminating line-of-sight projection and blending weakens the significance of such a feature. From Figure 8, it is also clear that the typical magnitudes of the innermost and peripheral SGB stars are characterized by an offset of $\sim 0.2$ mag.

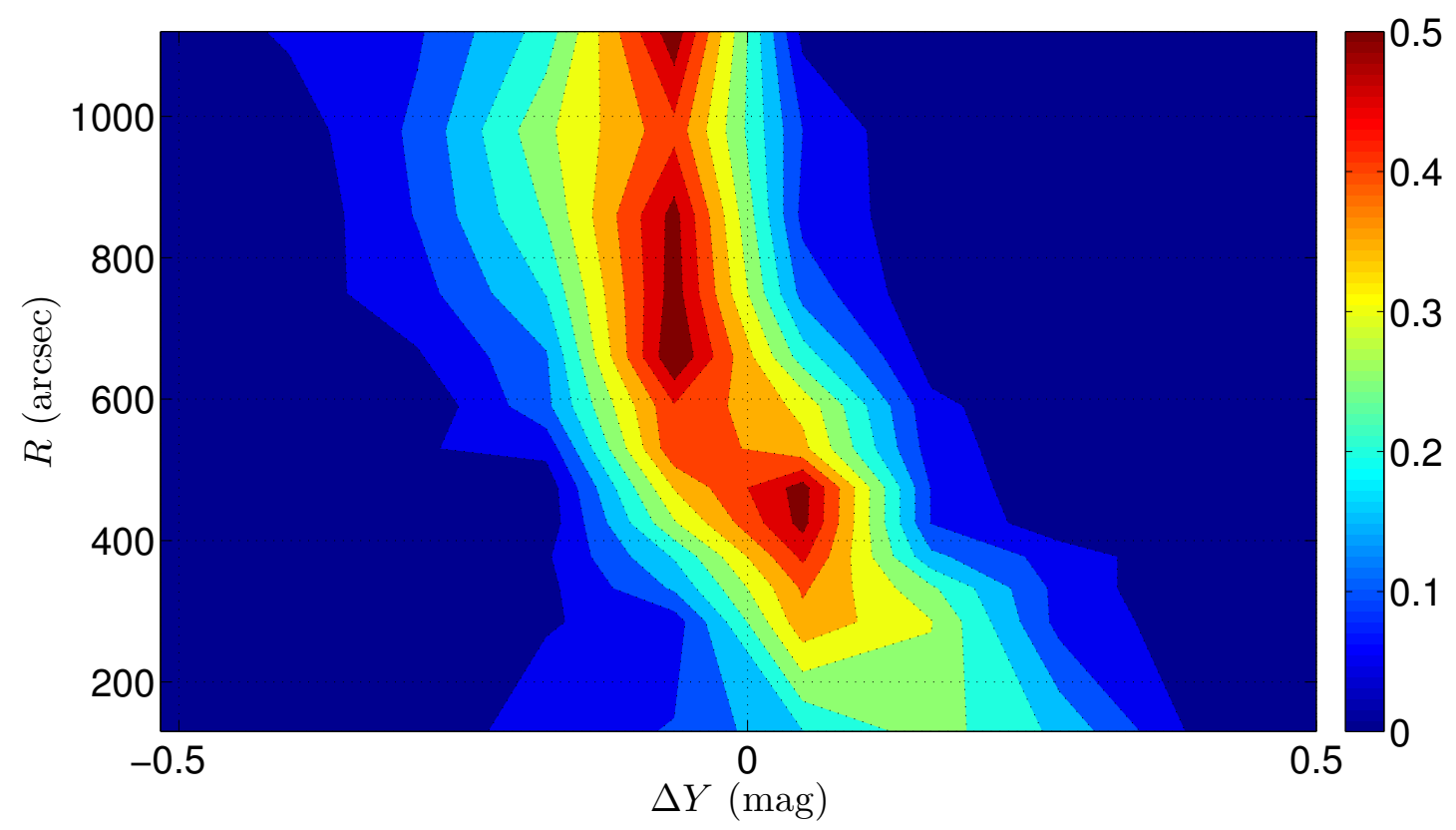

Fig. 8. - 2D contours of the SGB stars' probability distribution as a function of $\Delta Y$ and radius.

Note that SGB stars at $R \lesssim 150^{\prime \prime}$ cannot be detected because of crowding in the cluster center; the resulting stellar blends, in particular of the faint stellar wings of the PSF, cause an increase in the overall background level. This results in a very small completeness fraction for the innermost stellar sample, which clearly increases towards the cluster's periphery. In Figure 9 we display the local average completeness for stars with $15.4 \leq Y \leq 16.6$ mag, corresponding to the magnitude range of the SGB stars analyzed in this paper. Except for the innermost sample stars, all SGB stars analyzed here are located in regions where the sampling completeness is greater than $50 \%$. 


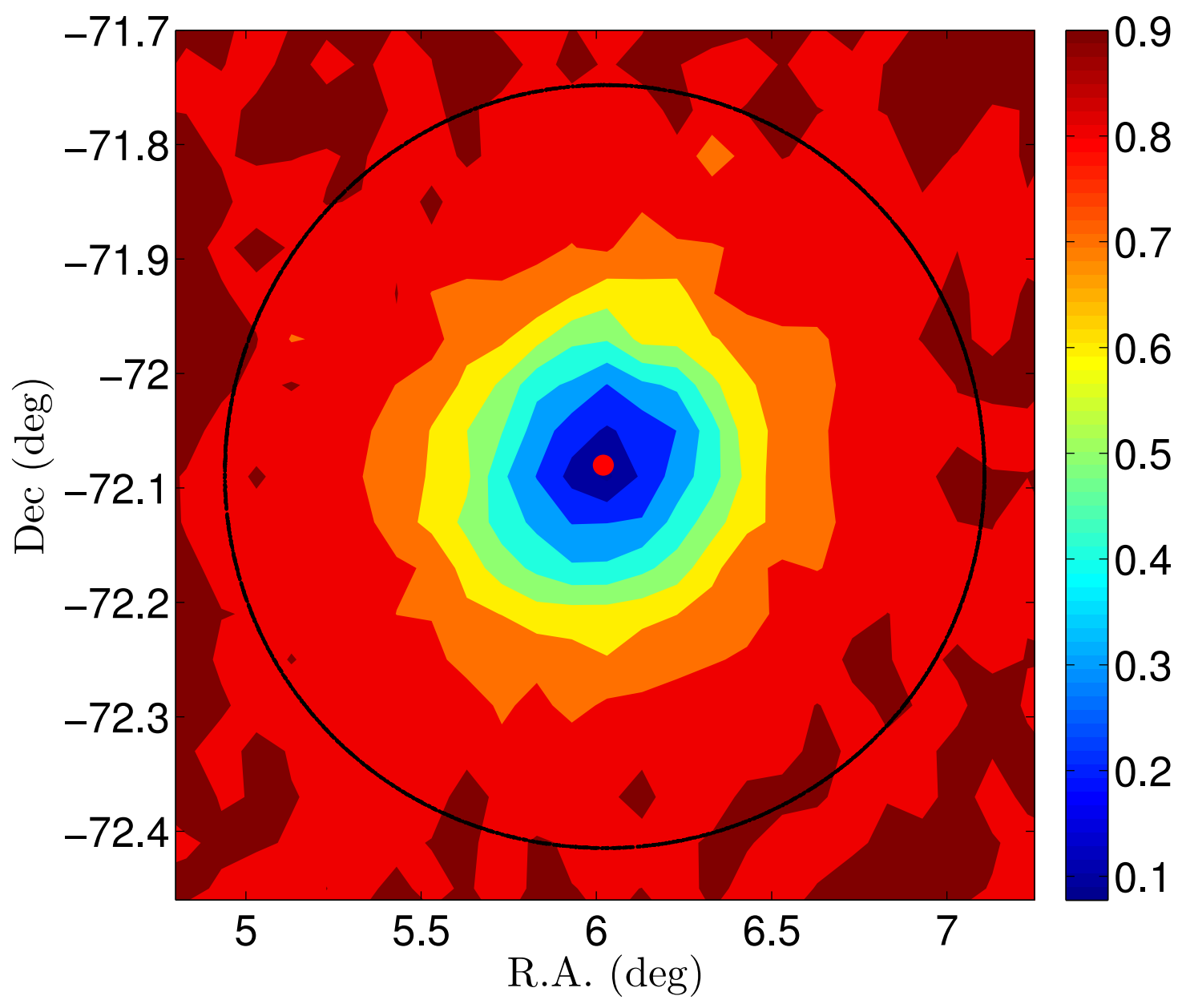

Fig. 9.- Completeness map for SGB stars characterized by $15.4 \leq Y \leq 16.6$ mag. The solid black circle indicates a radius of $R=1200^{\prime \prime}$. The cluster center is shown as a red solid bullet.

\subsection{Red-giant-branch stars}

Figure 3 reveals another striking feature in the form of a trend between RGB color and radius. Bluer RGB stars are located in the cluster's outskirts while the innermost RGB stars are clearly redder and, hence, cooler. This trend is particularly apparent for stars at the bottom of the RGB. Again, we defined a box that included the majority of stars at the bottom of the RGB, covering the region $Y \in[14.3,15.8] \mathrm{mag},\left(Y-K_{\mathrm{s}}\right) \in[0.7,1.2]$ mag. We removed 1352 stars with photometric errors greater than 0.015 mag, which guarantees that the broadening of the RGB cannot be only caused by photometric uncertainties. As 
for the SGB stars, we adopted the cluster-wide ridge line as benchmark and calculated the color deviation of the selected RGB sample stars from the corresponding benchmark color, $\Delta\left(Y-K_{\mathrm{s}}\right)=\left(Y-K_{\mathrm{s}}\right)_{\mathrm{RGB}}-\left(Y-K_{\mathrm{s}}\right)_{\text {iso }}$. We used a Gaussian-like profile to fit the distribution of the color dispersion, characterized by a Gaussian $\sigma_{\Delta\left(Y-K_{\mathrm{s}}\right)}=0.045 \mathrm{mag}$. We will only consider the $1850 \mathrm{RGB}$ stars that are found within $3 \sigma=0.135$ mag of the ridge line, corresponding to $58 \%$ of the full RGB sample in the area used for our sample selection. Figure 10 shows the zoomed-in CMD of the cluster's RGB region.

We next proceeded with our analysis in a similar manner as for the SGB stars. We divided the distribution of all RGB stars into five radial bins, with boundaries at $260^{\prime \prime}, 370^{\prime \prime}$, $510^{\prime \prime}$, and $730^{\prime \prime}$, thus ensuring roughly equivalent numbers of stars (varying from 365 to 377 ) in each bin. We then calculated the $\Delta\left(Y-K_{\mathrm{s}}\right)$ probability distributions (normalized to the total numbers of stars in each bin), as shown in Figure 11. Again, the result shows that the RGB gradually becomes bluer when going from the cluster's inner regions to its outskirts, which indicates that the peripheral RGB stars are systematically hotter than their more centrally located counterparts. The inner region's RGB, especially for the $R \leq 260^{\prime \prime}$ sample, is quite dispersed compared to the RGB stars at larger radii. This is again most likely owing to contamination from projected RGB stars that are physically located at large(r) radii but seen along more central lines of sight. This blending effect may also cause a change in the photometric colors of the RGB stars, but because the RGB is rather vertical, blending will most likely predominantly cause a shift in magnitude.

In Figure 12 we compare the CMD of the innermost RGB sample $\left(R \leq 260^{\prime \prime}\right)$ with that drawn from the largest radii associated with the cluster $\left(R \in\left[730^{\prime \prime}, 1200^{\prime \prime}\right]\right)$. The behavior of the RGB stars is similar to that seen for the cluster's SGB stars: both RGB samples occupy clearly different loci in CMD space, with almost all peripheral RGB stars being bluer than the benchmark isochrone.

In Figure 13 we display the 2D contours of the probability distribution as a function of both $\Delta\left(Y-K_{\mathrm{s}}\right)$ and radius. It is clear that the average color and its dispersion gradually evolve from the cluster's outskirts to the inner regions. The CMD of the RGB stars located at large radii is narrower and bluer than that representative of the inner-sample RGB stars. The probability distribution shows a continuous ridge from the cluster's outer boundary to $R \sim 500^{\prime \prime}$, followed by a peak between $R=200^{\prime \prime}$ and $R=400^{\prime \prime}$ and a significant increase in the color dispersion to $\geq 0.1 \mathrm{mag}$ in the innermost regions. Such a large color dispersion cannot be only caused by an intrinsic photometric dispersion, given that we have constrained the photometric uncertainties (in magnitude) of all sample stars to $\leq 0.015$ mag. The observed width of $\Delta\left(Y-K_{\mathrm{s}}\right)$ is five times the allowed maximum photometric broadening due to magnitude errors. Again, we use the Monte Carlo method to estimate the contamination 


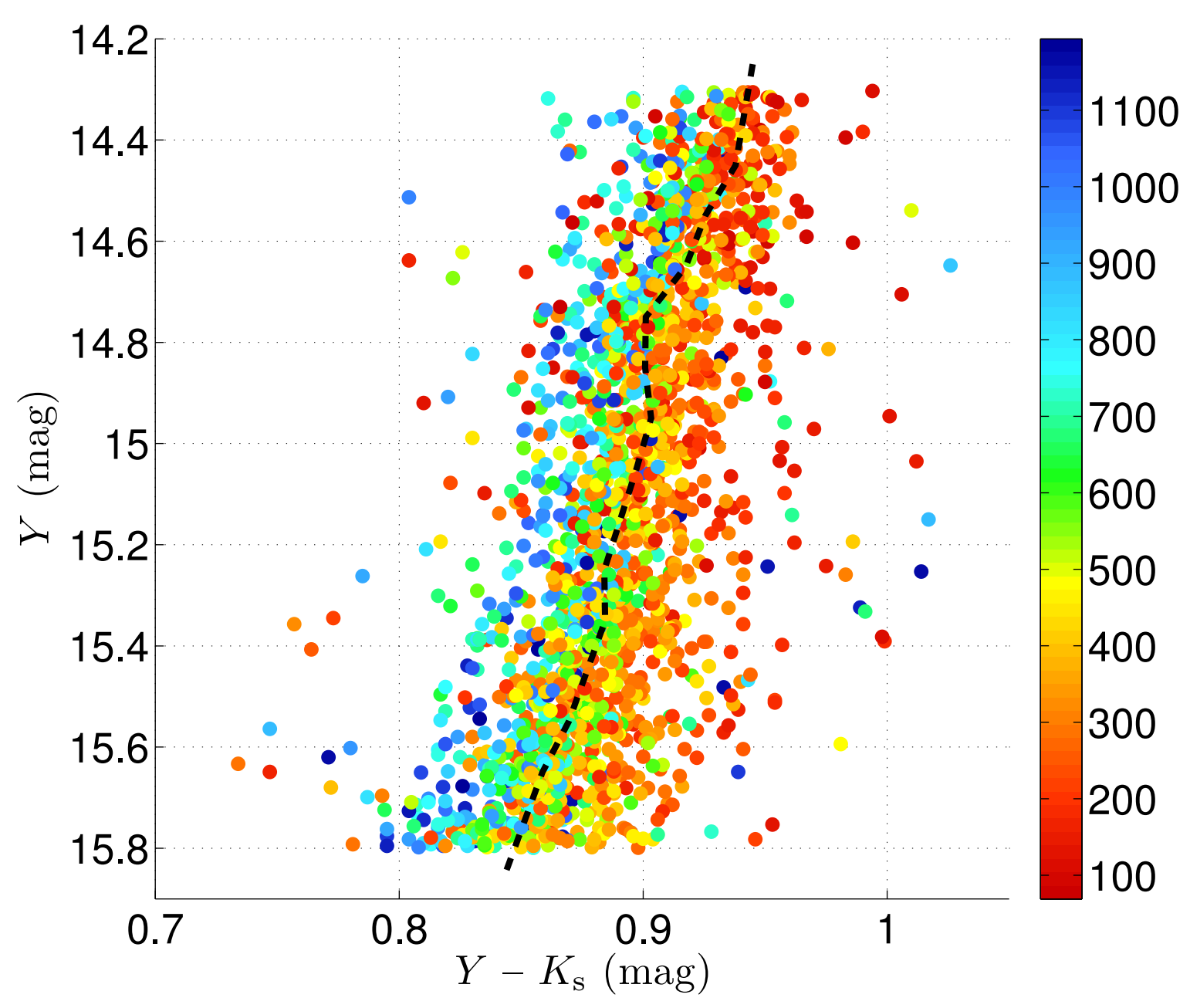

Fig. 10. - CMD of the adopted RGB region, showing only stars found within $3 \sigma=0.135$ mag of the isochrone's ridgeline, where $\sigma$ represents the color spread, adopting a Gaussian fitting function. The color scale represents distance to the cluster center. Because of sampling incompleteness in the cluster core, stellar photometry is only robustly available for radii beyond $R \sim 70^{\prime \prime}$.

fraction due to projection for all radial ranges (see Table 1).

Because of the higher levels of sampling incompleteness in the cluster core, the minimum radius employed in our analysis of the RGB's stellar population is $R=70^{\prime \prime}$. Figure 14 shows the $2 \mathrm{D}$ completeness map for stars with $14.2 \leq Y \leq 16.0$ mag. For $R \gtrsim 70^{\prime \prime}$, all RGB stars used for our analysis are located in regions where the completeness fractions are greater than 

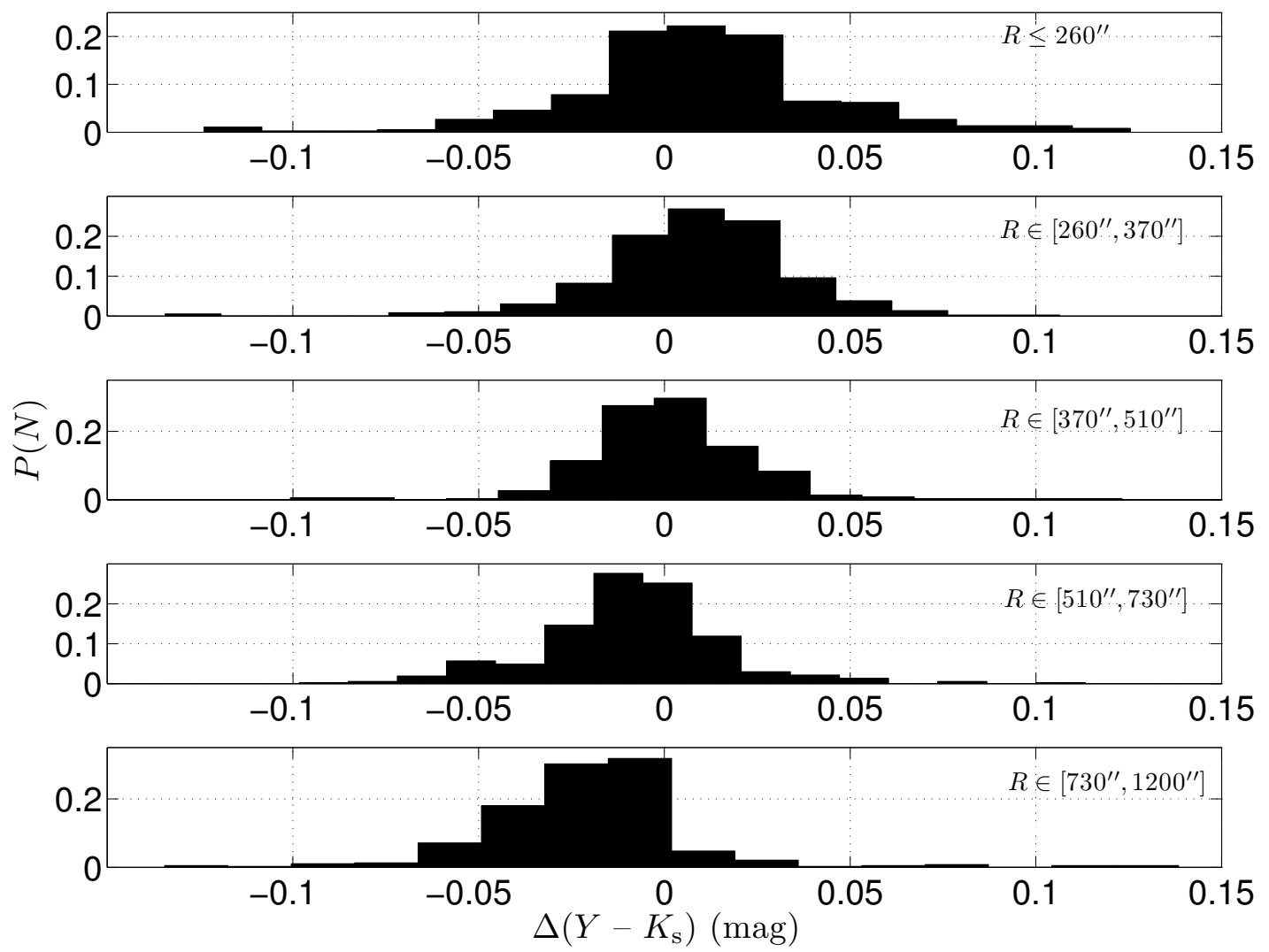

Fig. 11. - Probability distribution of $\Delta\left(Y-K_{\mathrm{s}}\right)$ as a function of radius for RGB stars in 47 Tuc. From top to bottom, the panels represent radial bins from the cluster's inner regions to its outskirts.

$50 \%$.

\section{Physical implications}

Before we discuss the possible physical implications of the results presented in the previous section, we first rule out differential reddening as the dominant cause of the observed trends for the SGB and RGB stars in 47 Tuc. We obtained the extinction map of the 47 Tuc area from the IRAS/DIRBE database provided by the NASA/IPAC Infrared Science Archive (cf. Schlegel et al. 1998; Schlafly \& Finkbeiner 2011) (see also Qing \& Sneden 2010). Although the database's native spatial resolution is on the order of an arcminute, 


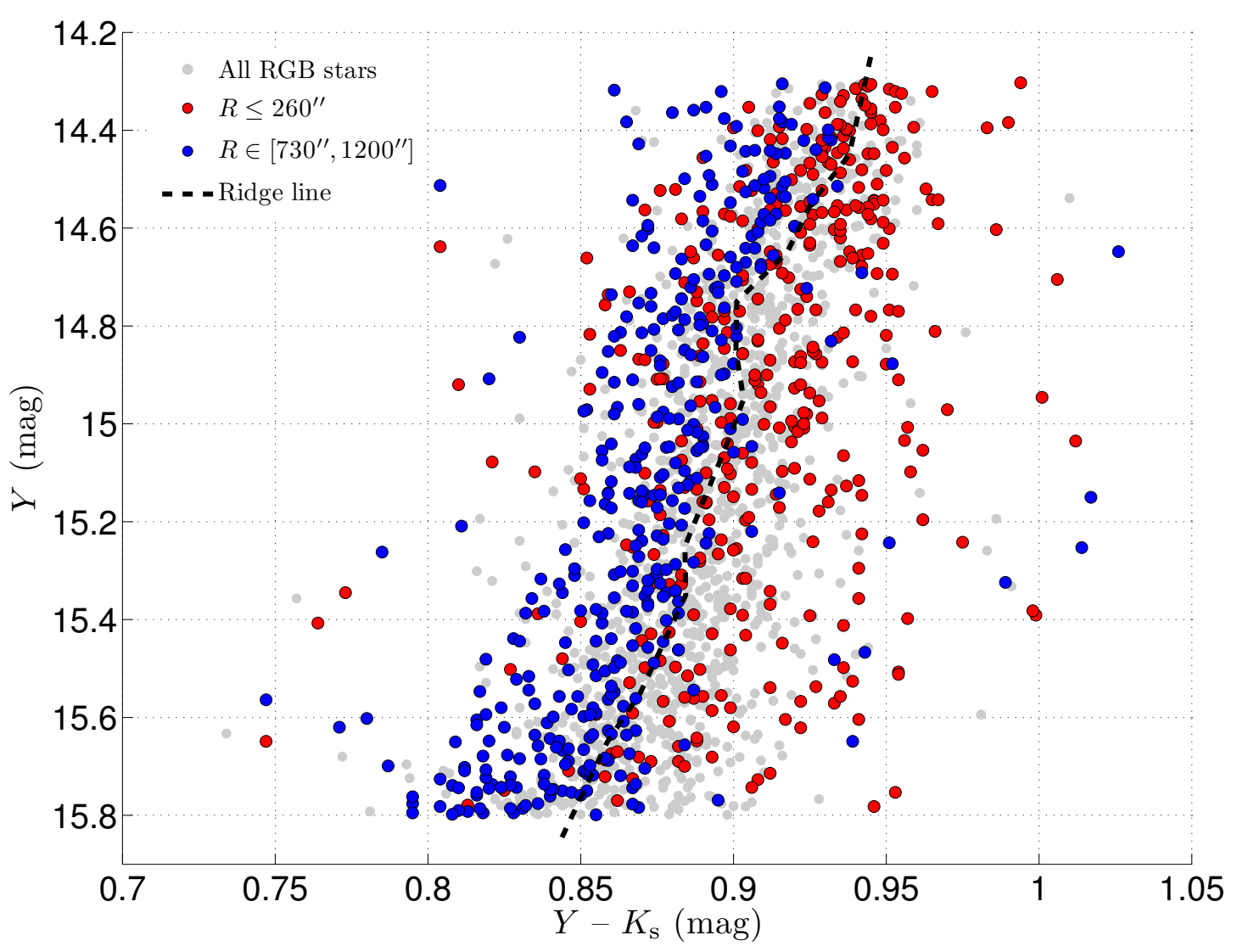

Fig. 12. - CMD comparison of the innermost RGB stars ( $R \leq 260^{\prime \prime}$ : red solid bullets) with the outermost cluster RGB stars $\left(R \in\left[730^{\prime \prime}, 1200^{\prime \prime}\right]\right.$ : blue solid bullets). Smaller grey bullets represent the full RGB sample.

all details within the cluster region have been smoothed: we adopt a radius for the cluster region covered by our observations of $20^{\prime}$, while the radial bins used for the analysis are only slightly smaller than $5^{\prime}$. This hence implies that we could potentially resolve any dust substructures within the cluster, provided that they exist. Note that our reddening corrections may also be applicable to even smaller spatial regions, because the spread on the lower RGB is larger than that higher up on the RGB; extinction effects cannot be used as discriminant in this case. We measured the extinction distribution from the cluster center to $R=1200^{\prime \prime}$ in radial steps of $5^{\prime}$. We found that the average extinction in this region varies from $E(B-V) \simeq 0.027$ mag to $0.028 \mathrm{mag}$; the corresponding extinction at NIR wavelengths is therefore negligible and unimportant in the context of the observed broadening of both the SGB and RGB in 


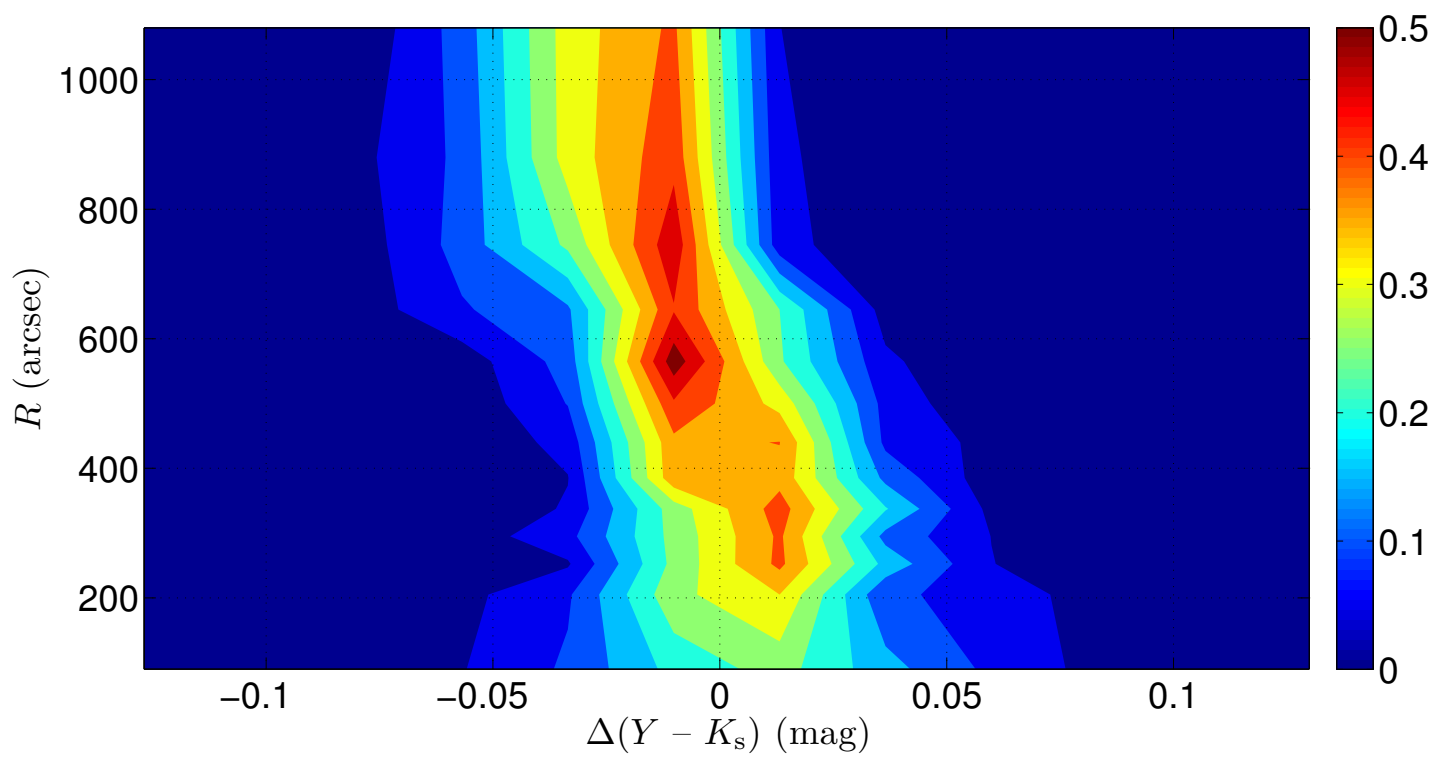

Fig. 13. - 2D contours of the RGB stars' probability distribution as a function of $\Delta\left(Y-K_{\mathrm{s}}\right)$ and radius.

the 47 Tuc CMD. This result is also consistent with that published by Salaris et al. (2007).

In addition, Momany et al. (2012) and Boyer et al. (2010) conclude that there is no evidence that RGB stars in 47 Tuc produce dust. These arguments are supported by the results of Bonatto et al. (2013), who map the differential reddening in 66 GCs (including 47 Tuc; their fig. 4) and conclude that the main source of differential reddening is interstellar rather than intracluster dust.

Milone et al. (2012c) used helium and nitrogen enhancements to reproduce the observed double MS in 47 Tuc, implying that the second generation of stars formed from material that had been polluted by the first generation. Additional evidence in support of the notion that GCs should contain N-rich but COdepleted second-generation stars is found in the GC NGC 6656 (M22), which has been confirmed to host two groups of stars characterized by different $\mathrm{C}+\mathrm{N}+\mathrm{O}$ abundances (Marino et al. 2011, 2012). Di Criscienzo et al. (2010) also suggested that variations in $\mathrm{C}+\mathrm{N}+\mathrm{O}$ abundances may be responsible for a broadening of GC SGB features, because differences among CNO-group elements will change the strength of their compound absorption lines (e.g., OH, NH, CN, and CH). Optical filters, particularly those covering wavelengths between $3500 \AA$ and 4500 $\AA$, are sensitive to these absorption lines (see Milone et al. 2012c, their figure 11). Variations in CNO abundances may hence introduce an additional broadening 


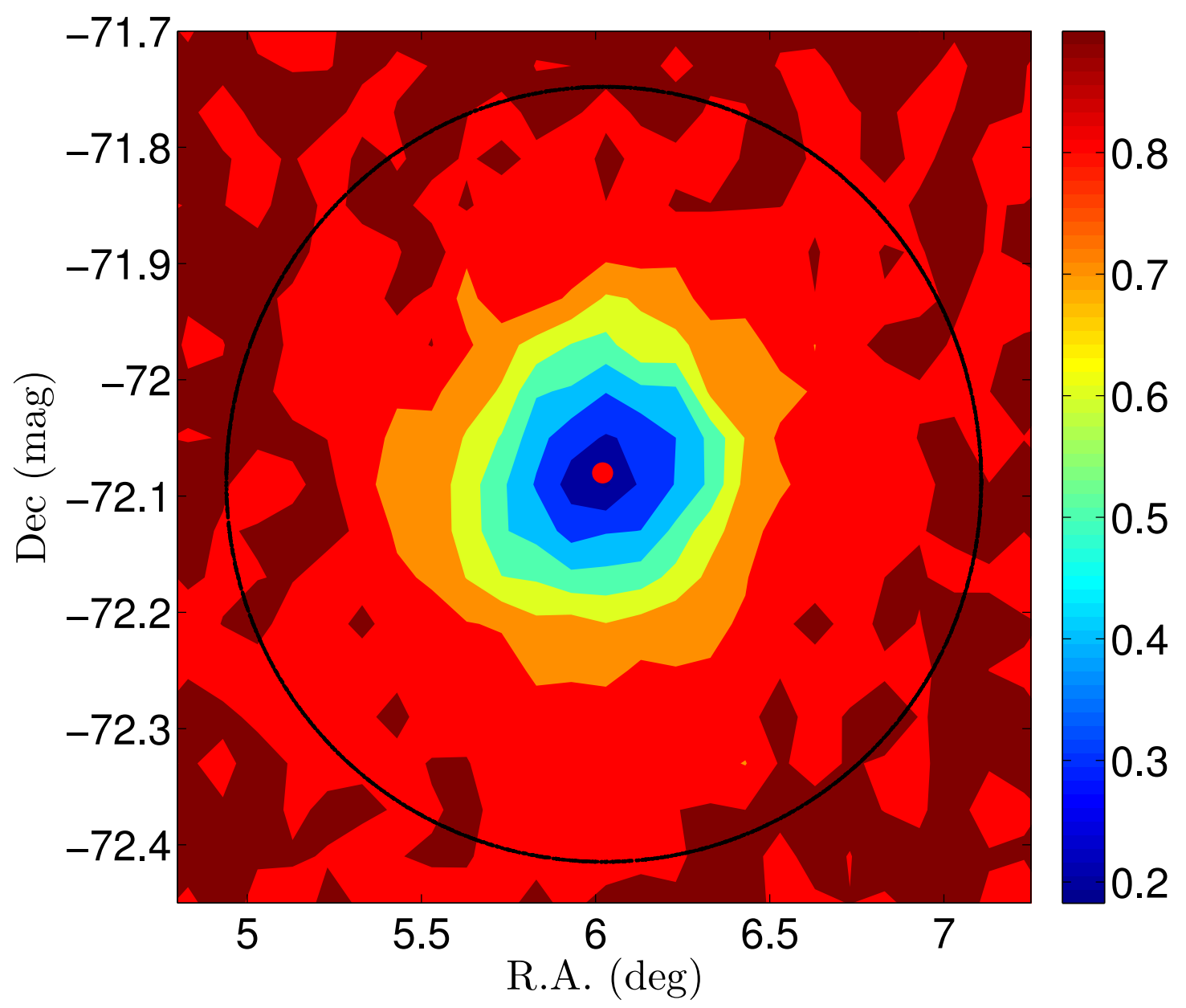

Fig. 14. - Completeness map for RGB stars characterized by $14.2 \leq Y \leq 16.0$ mag. The solid black circle indicates a radius of $R=1200^{\prime \prime}$. The cluster center is shown as a red solid bullet.

of CMD features observed in the optical wavelength range. However, our data have all been obtained in the NIR $Y$ and $K_{\mathrm{s}}$ filters, which are characterized by effective wavelengths of $1.02 \mu \mathrm{m}$ and $2.15 \mu \mathrm{m}$, respectively. It is, hence, impossible that CNO differences would be solely responsible for a significant broadening of the SGB and RGB features in the 47 Tuc $\left(Y, Y-K_{\mathrm{s}}\right)$ CMD.

Ejecta from stars of a previous stellar generation will contaminate the second generation, thus causing $\alpha$ enhancement. However, previous analyses (cf. Carretta et al. 2004) have not reported any significant $[\alpha / \mathrm{Fe}]$ dispersion in 47 Tuc. We nevertheless explored the effects 
of possible $[\alpha / \mathrm{Fe}]$ variations. We adopted PGPUC isochrones characterized by $[\alpha / \mathrm{Fe}]=0.0$ to 0.3 dex for comparison, but found that $[\alpha / \mathrm{Fe}]$ variations do not significantly change the isochrone's morphology in either the SGB or RGB phases. Since for these stellar evolutionary phases the effects of helium and $[\alpha / \mathrm{Fe}]$ differences are both minor to negligible, we will henceforth adopt $\mathrm{Y}=0.26$ and $[\alpha / \mathrm{Fe}]=0.30 \mathrm{dex}$.

The most straightforward explanation of the observed radial trends in the magnitudes of the cluster's SGB stars and the colors of its RGB stars is the presence of multiple stellar populations. Indeed, Milone et al. (2012c) investigated 47 Tuc's apparent triple MSs and found a significant helium dispersion, $\mathrm{Y}=0.256-0.288$ (their table 2 and figure 10 , based on the assumption that the presence of a dispersion in helium content is the only cause of the MS split). This result is consistent with that of Nataf et al. (2011), which is based on their analysis of RGB-bump stars and HB stars $(\mathrm{Y}=0.25-0.28)$. Note that these authors did not base their conclusions on the properties of the cluster's SGB and RGB stars, the stellar phases we concentrate on here.

In addition to this variation in the cluster's helium abundance, a dispersion in stellar metallicities could also contribute to the observed broadening of the 47 Tuc SGB and RGB. A significant number of studies have explored this issue, going back to at least Brown \& Wallerstein (1992), who investigated four giant-branch stars in the cluster. They found minimum and maximum $[\mathrm{Fe} / \mathbf{H}]$ abundances of -0.88 dex and -0.69 dex, respectively, or $Z$ ranging from $\sim 0.0020$ to 0.0031. Subsequently, Carretta \& Gratton (1997) obtained spectroscopic observations of three 47 Tuc RGB stars and derived a mean $[\mathrm{Fe} / \mathrm{H}]$ abundance of $-0.70 \pm 0.03(Z=\mathbf{0 . 0 0 2 8}-\mathbf{0 . 0 0 3 3})$. Given that studies based on such small numbers of stars cannot be used to infer statistically robust results, we will instead focus on a number of more modern studies. Carretta et al. (2004) analyzed highdispersion spectra of three dwarfs and nine subgiants; they confirmed an $[\mathrm{Fe} / \mathrm{H}]$ range from -0.59 dex to $-0.78 \mathrm{dex}(Z=0.0026-0.0041)$. Koch \& McWilliam. (2008) investigated eight RGB and one MSTO stars, and determine that the most representative metallicity, $[\mathrm{Fe} / \mathrm{H}]=-0.76 \pm 0.05 \mathrm{dex}$, which is equivalent to a $Z$ range from 0.0024 to 0.0030 .

We point out that, compared with spectroscopic studies, a number of recent articles used photometric measurements to derive a higher overall metallicity for the cluster, as well as a larger dispersion. Salaris et al. (2007) used the BaSTI models to analyze their photometric observations, yielding $Z=0.004 \pm 0.001$. (They conclude that $[\mathrm{Fe} / \mathrm{H}]=-0.7 \pm 0.1 \mathrm{dex}$, but this determination is based on $Z_{\odot} \sim 0.02$; they also find that the corresponding $Z=0.008$, which we suspect is 
a clerical error.) Nataf et al. (2011) analyzed the 47 Tuc RGB bump stars and concluded that they had to assume a model with $[\mathbf{M} / \mathbf{H}]=-0.50$ or -0.52 dex (corresponding to $[\mathrm{Fe} / \mathrm{H}]=-0.53$ or -0.55 dex for $[\mathrm{M} / \mathrm{H}]=0.95[\mathrm{Fe} / \mathrm{H}]$ ) to fit their observations. Anderson et al. (2009) analyzed the spread among the SGB stars. They suggest that if metallicity is the only driver of the observed spread, a metallicity dispersion of $0.10 \mathrm{dex}$ is implied. These differences compared with spectroscopic metallicity determinations may well be owing to an inherent selection bias that predominantly affects spectroscopic observations. After all, to adequately resolve individual stellar spectra, one should try to avoid crowded environments and, hence, select candidate stars that are located far from the cluster center. For instance, Carretta et al. (2004) selected their sample stars at distances in excess of $800^{\prime \prime}$ from the 47 Tuc core. To reconcile the differences between the photometric and spectroscopic metallicity determinations in the context of the analysis presented in this article, we adopt $Z=0.0041$ from Carretta et al. (2004) as the typical 47 Tuc metallicity, but we assume that the dispersion in metallicity is represented by that implied by the photometric analyses, i.e., $\Delta_{[\mathrm{Fe} / \mathrm{H}]} \sim 0.10$ dex (corresponding to a full metallicity range given by $Z=0.0033-\mathbf{0 . 0 0 5 1 )}$.

We used a Monte Carlo method to mimic the distribution in CMD space of the observed SGB and RGB stars, based on adoption of two suitable 'bracketing' isochrones. We follow the usual assumption that the helium-rich generation of stars should also be metallicity enhanced and adopt the highest-dispersion metallicity range from the literature for our model isochrones, i.e., $Z=0.0033-0.0051$ and $Y=0.25-0.28$. The helium and metallicity abundances must be varied only between these values. To generate a physically realistic broadening of the CMD, we also considered the possible presence of an age dispersion among the cluster's member stars. At the age of 47 Tuc, even a very small age dispersion represents a long time span, which may be enough for the brightest first-generation stars to evolve to late evolutionary stages. Previous studies have suggested a possible age dispersion among 47 Tuc stars of order 1 Gyr $(\sim 12-13$ Gyr; see Di Criscienzo et al. 2010). Gratton et al. (2003) found a model-dependent best-fitting age for 47 Tuc of either 11.2 \pm 1.1 Gyr or 10.8 \pm 1.1 Gyr, while Zoccali et al. (2001) determined an age of $13 \pm 2.5$ Gyr. Some recent papers have reported smaller possible age dispersions, with a typical age span of $12.8 \pm 0.6$ Gyr (Marín-Franch et al. 2009), $12.75 \pm 0.5$ Gyr (Dotter et al. 2010), or even 11.75 \pm 0.25 Gyr (Vandenberg et al. 2013). We hence conservatively select a minimum age difference of 0.25 Gyr for our model, e.g., $12.50 \pm 0.25$ Gyr. We thus generate an artificial 47 Tuc 
CMD defined by $\mathrm{Y}=0.25-0.28, Z=0.0033-0.0051$, and an age range spanning from 12.25 Gyr to 12.75 Gyr. In Figure 15 we display the corresponding range in best-fitting isochrones.

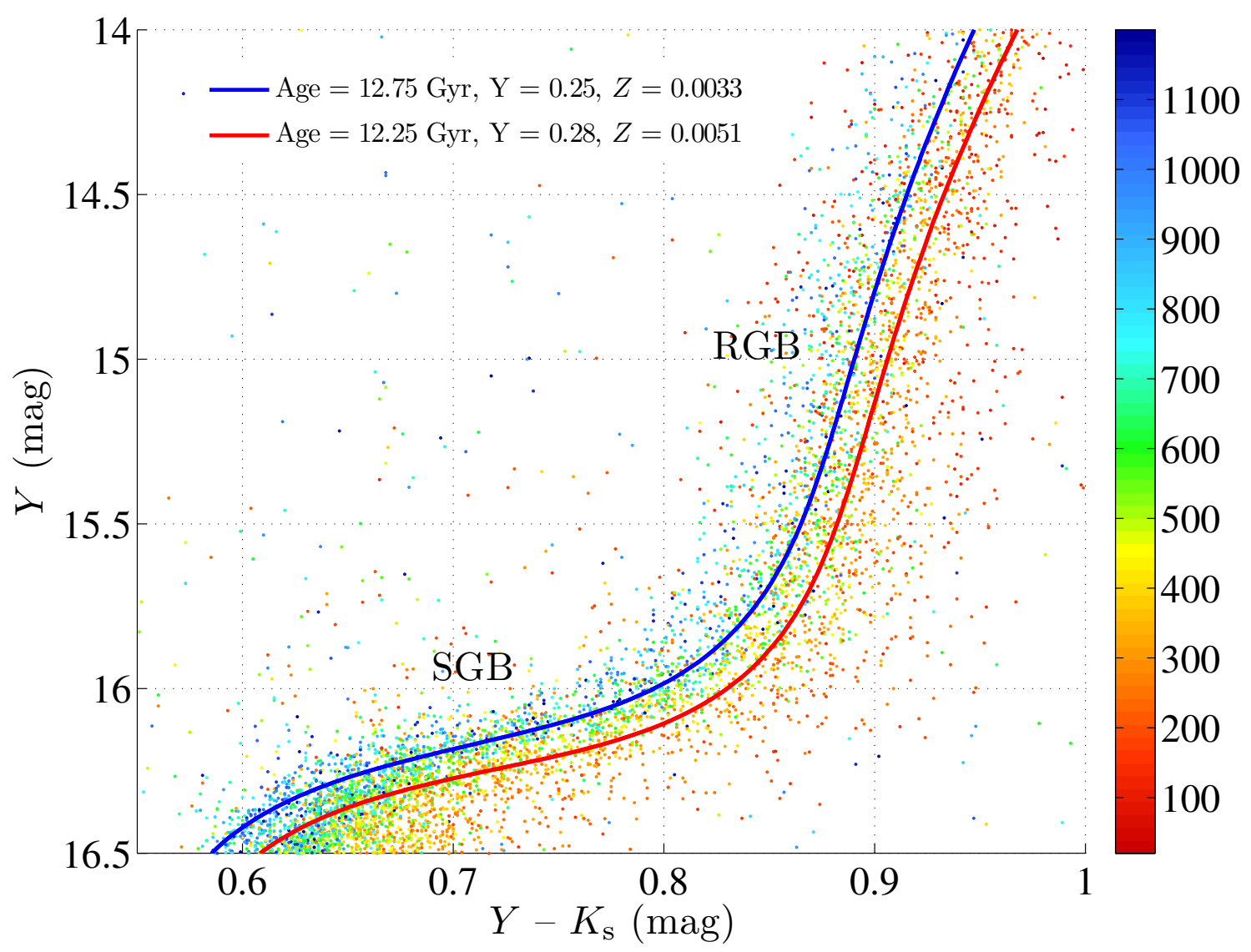

Fig. 15. - Isochrone fits of a young, helium-rich, and metal-rich model (red line), as well as of an old, helium-poor, and metal-poor model (blue line). The color scale represents the stars' distances to the adopted cluster center (in arcsec).

If we only mimic stars with photometric errors of up to $0.015 \mathrm{mag}$, we cannot reproduce the observations. Once again, this confirms that the observed SGB and RGB broadening is more likely caused by a dispersion in stellar generations and not solely by photometric uncertainties. Note that the typical $1 \sigma_{\Delta Y}$ and $1 \sigma_{\Delta\left(Y-K_{\mathrm{s}}\right)}$ dispersions of the SGB and RGB stars are $0.18 \mathrm{mag}$ and $0.045 \mathrm{mag}$, respectively (see the captions of Figures 5 and 10). The large, observed dispersion is owing to a combination of photometric uncertainties and a dispersion in stellar-generation properties (age, helium abundance, and metallicity). However, 
to avoid complications associated with adopting a full age range, we still assume that the bulk of the cluster stars can be adequately described by two separate generations of stars (but see below), although we relax their dispersions to cover $\mathbf{0 . 5} \sigma_{\Delta Y}=0.090 \mathrm{mag}$ and $\mathbf{0 . 5} \sigma_{\Delta\left(Y-K_{\mathrm{s}}\right)}=0.022 \mathrm{mag}$ for the simulated SGB and RGB stars, respectively. This still reproduces two apparent branches in the CMD. In Figures 16 and 17 we display the simulated CMDs for the SGB and RGB stars (right-hand panels), compared with the real observations (left-hand panels). The top two panels represent the overall comparison, while the bottom two panels only indicate the observed inner- and outermost samples, as well as their simulated counterparts. The simulated SGB and RGB samples contain the same numbers of stars as the observations. We adopt a flat magnitude distribution, because the magnitude range covered by the SGB stars is rather narrow, while for the RGB stars we are only concerned with their color dispersion. Hence, any difference in the luminosity function will not affect our RGB-based analysis. We also confirmed that the observed luminosity function pertaining to our selected sample of RGB stars is close to flat.

Instead of checking the performance of our fits by eye, we quantify the similarity between the observed and simulated CMDs using $\chi^{2}$ minimization. At a given radius, we assume that the simulated CMD contains a certain fraction of first-generation stars, $f_{\mathrm{fg}}$. The remainder of the stellar population at that radius then follows the second-generation isochrone, which hence is characterized by a fraction $f_{\mathrm{sg}}=1-f_{\mathrm{fg}}$. We then use the adopted ridge line (see Figures 5 and 10) to calculate the probability distributions of $\Delta Y$ for the simulated SGB stars, and of $\Delta\left(Y-K_{\mathrm{s}}\right)$ for the simulated RGB stars, similarly to what we did for the observations in Figures 6 and 11. As a function of radius, we then calculate the corresponding $\chi^{2}$ value,

$$
\chi^{2}=\sum_{n} \frac{\left(N^{\prime}-N\right)^{2}}{N},
$$

where $N^{\prime}(N)$ indicates the simulated (observed) number of stars in different $\Delta Y$ or $\Delta\left(Y-K_{\mathrm{s}}\right)$ bins (for SGB and RGB stars, respectively), and $n$ represents the number of bins. The $\chi^{2}$ value indicates the level of similarity between the simulated and observed CMDs. We vary $f_{\mathrm{fg}}$ from $5 \%$ to $95 \%$ and determine the minimum $\chi^{2}$ value for each input $f_{\mathrm{fg}}$. We find that the use of a parabolic function can approximate the $\chi^{2}\left(f_{\mathrm{fg}}\right)$ distribution very well: it will yield both the global minimum $\chi^{2}$ value (and, hence, the best-fitting $f_{\mathrm{fg}}$ ) and its $1 \sigma$ uncertainty (see also de Grijs et al. 2013). The latter corresponds to the difference between $\chi_{\min }^{2}$ and $\chi_{\min }^{2}+1$ (Avni 1976; Wall 1996). We repeat each calculation 100 times and 


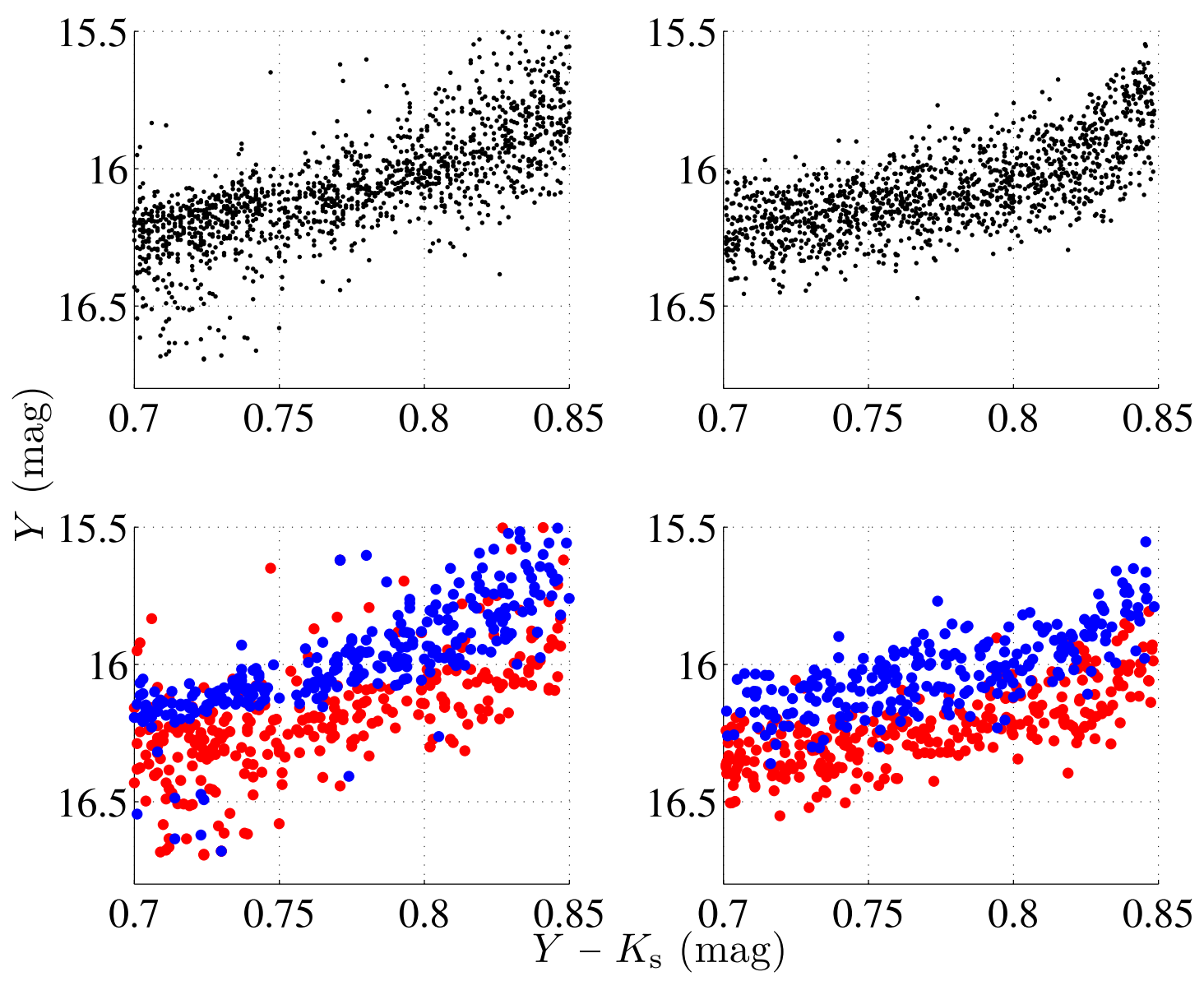

Fig. 16.- Top left: Observed overall distribution of SGB stars. Top right: Simulated SGB stars. Bottom left: Observed innermost SGB stars $\left(R \leq 340^{\prime \prime}\right.$ : blue solid bullets) and outermost SGB stars $\left(R \in\left[730^{\prime \prime}, 1200^{\prime \prime}\right]\right.$ : red slid bullets). Bottom right: Simulated innermost SGB and outermost SGB stars.

record the average value as the corresponding $\chi^{2}$. We thus eliminate random scatter in the $\chi^{2}$ values and obtain a smooth $\chi^{2}$ curve as a function of $f_{\mathrm{fg}}$.

In Figures 18 and 19, we show the calculated $\chi^{2}$ distributions for different input fractions of $f_{\mathrm{fg}}$, as well as the best-fitting parabolic curves, for the SGB and RGB samples, respectively. From top to bottom, the panels display the results for the innermost to outermost radial samples; the arrows represent the best-fitting $f_{\mathrm{fg}}$ fractions. For both the SGB and RGB stars, the best-fitting $f_{\mathrm{fg}}$ fractions for the outermost samples are $100 \%$. This indicate that at those radii, 


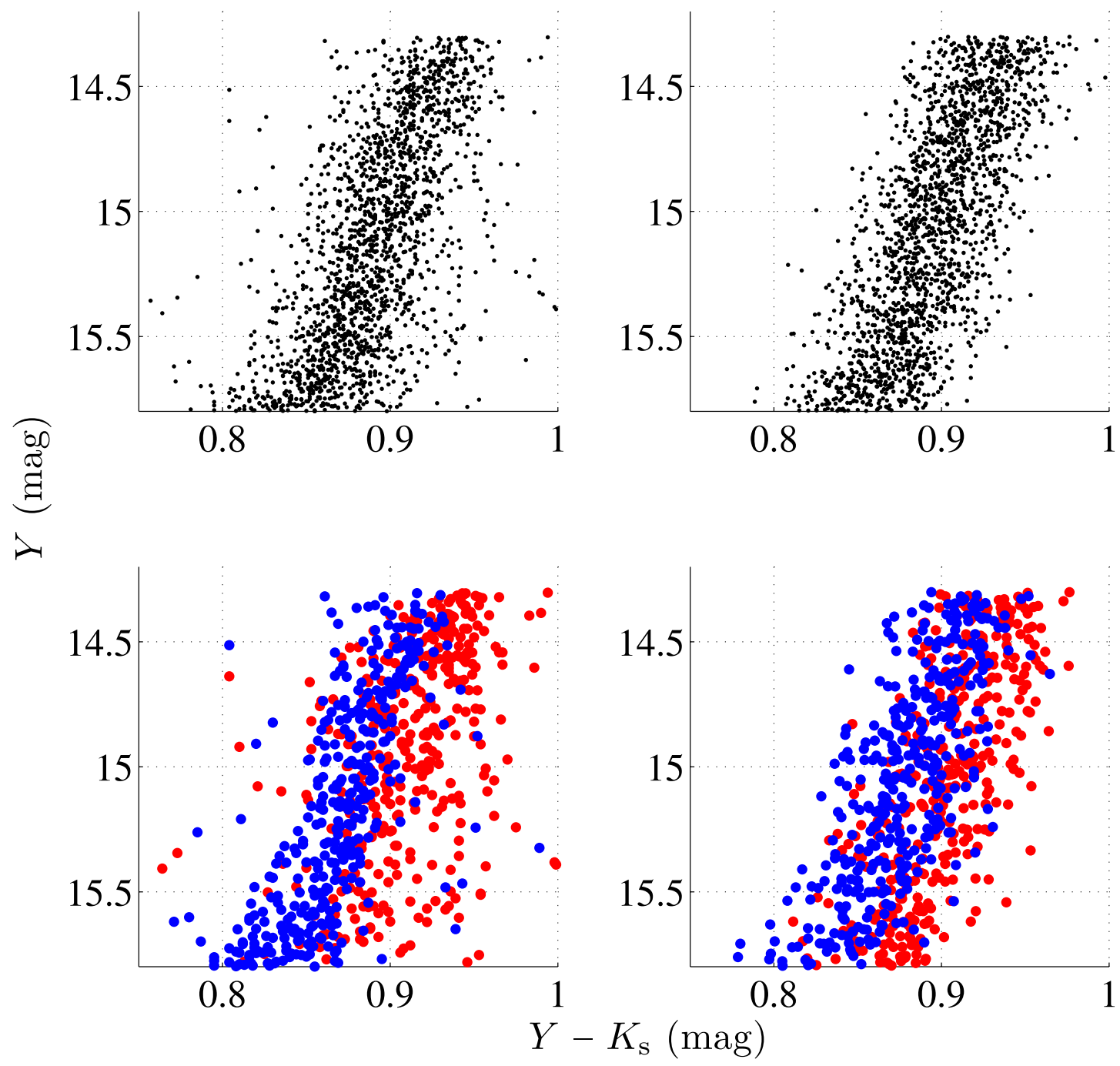

Fig. 17. - As Figure 16, but for RGB stars. The innermost sample contains stars with $R \leq 260^{\prime \prime}$, while the outermost sample covers $R \in\left[800^{\prime \prime}, 1200^{\prime \prime}\right]$.

the composition of 47 Tuc is still close to that expected for a simple stellar population. It is also clear that for both the SGB and RGB stars, the firstgeneration stars only occupy a small fraction in the innermost region, but that this fraction increases significantly toward the cluster's outskirts, reaching unity at the adopted outer boundary, $R=1200^{\prime \prime}$.

In Figure 20 we display the best-fitting $f_{\mathrm{fg}}$ fraction as a function of radius, 
for both the SGB stars (blue line) and their RGB counterparts (red line). The error bars indicate the $1 \sigma$ statistical uncertainties, which are all less than $5.5 \%$. The innermost sample is clearly only composed of a very small fraction of firstgeneration stars, which implies that most member stars should belong to the second generation. On the other hand, it appears that the outermost region can still be well described by a simple stellar population. In addition, even if we investigate the SGB and RGB stars separately, both exhibit consistent trends in $f_{\text {fg }}$ from $R \sim 400^{\prime \prime}$ to $R \sim 600^{\prime \prime}$. This strongly implies that they form intrinsically uniform samples: both their inner- and outermost members share a continuous track in the CMD. However, compared with the SGB stars in the innermost region, the RGB stars in the same region show a relatively large $f_{\mathrm{fg}}$ fraction, which may be caused by the large projected contamination and small completeness in this region. We summarize these statements quantitatively in Table 2.

Table 2: Best-fitting $f_{\mathrm{fg}}$ fractions as a function of radius.

\begin{tabular}{l|c||l|c}
\hline \hline SGB stars & $f_{\text {fg }}$ & RGB stars & $f_{\text {fg }}$ \\
\hline \hline$R \leq 340^{\prime \prime}$ & $9.02 \%$ & $R \leq 260^{\prime \prime}$ & $21.50 \%$ \\
$R \in\left[340^{\prime \prime}, 450^{\prime \prime}\right]$ & $24.50 \%$ & $R \in\left[260^{\prime \prime}, 370^{\prime \prime}\right]$ & $11.10 \%$ \\
$R \in\left[450^{\prime \prime}, 590^{\prime \prime}\right]$ & $55.57 \%$ & $R \in\left[370^{\prime \prime}, 510^{\prime \prime}\right]$ & $40.20 \%$ \\
$R \in\left[590^{\prime \prime}, 800^{\prime \prime}\right]$ & $94.31 \%$ & $R \in\left[510^{\prime \prime}, 730^{\prime \prime}\right]$ & $76.00 \%$ \\
$R \in\left[590^{\prime \prime}, 800^{\prime \prime}\right]$ & $100 \%$ & $R \in\left[510^{\prime \prime}, 730^{\prime \prime}\right]$ & $100 \%$ \\
\hline \hline
\end{tabular}

In summary, the observed distributions of SGB and RGB stars in the 47 Tuc CMD can best be explained if the cluster's SGB and RGB stars in the outer regions are both heliumand metal-poor, while their more centrally located counterparts are helium- and metal-rich.

Our results decisively confirm the presence of second-generation stars in 47 Tuc. Milone et al. (2012c, their figure 33) calculated the number frequency of red RGB stars (which they refer to as "RGBb"), which increases from the cluster's periphery (RGBb/RGB $\sim 60 \%$ ) to its central region $(\mathrm{RGBb} / \mathrm{RGB} \sim 80 \%$ ), while the average RGB color and its dispersion remain roughly constant. A similar result is presented by Cordero et al. (2014), who found that the fraction of RGBb stars increases from $\sim 40 \%$ to $\sim 90 \%$ from the cluster's outskirts to its central regions. Our analysis yields a fraction of $5-\mathbf{1 0 \%}$ in the outermost region to $\sim 90 \%$ in the cluster core. This is a similar although even more significant trend as that reported by Milone et al. (2012c). Our result is also consistent with the $N$-body simulations of Vesperini et al. (2013).

This scenario is supported by the spatial distributions of the SGB and RGB stars. The 
second generation stars would more likely have formed within the denser core region of the cluster, and their clearly different distributions suggest that the second-generation stars may originally have been dominated (in terms of stellar numbers) by first-generation stars, but that most first-generation stars subsequently somehow escaped from the cluster. This may be owing to expansion of the cluster triggered by either Type II supernovae (Decressin et al. 2008; D'Ercole et al. 2008) or tidal stripping (Milone et al. 2012c), which eventually led to a system with second-generation stars preferentially distributed closer to the cluster center.

The analysis presented in this article is the first investigation of the radial behavior of the multiple populations of SGB stars in 47 Tuc. The suggested helium-abundance dispersion of $\Delta Y=0.03$ fully concurs with that derived by Anderson et al. (2009), $\Delta Y=0.026$. Figure 8 shows that this helium enhancement may be most significant near $R \sim 500^{\prime \prime}$, where one can discern two clear peaks on either side of this radius. Our simulation results also confirm this result. For both SGB and RGB stars, the $f_{\text {fg }}$ fractions increase significantly from $R \sim 400^{\prime \prime}$ to $R \sim 600^{\prime \prime}$, exactly covering the locations of these two peaks. This indicates that pollution by a second generation of stars becomes apparent at these radii. The discovery of a population of more centrally concentrated helium-rich stars is also consistent with the analysis of Bekki (2011), Nataf et al. (2011), and Ventura et al. (2014). The derived enhanced metallicity implies an origin related to the ejecta of massive stars (Decressin et al. 2007; de Mink et al. 2009; Ventura \& D'Antona 2009; Cordero et al. 2014).

\section{Conclusions}

We have presented a deep, wide-area, NIR CMD of - and the corresponding spatial distribution of the SGB and RGB stars in -47 Tuc, obtained with the VISTA telescope. Apparent differences are seen between SGB and RGB stars in the cluster's outskirts compared with its inner regions. The peripheral SGB stars are systematically brighter and define a narrower CMD feature than the SGB stars in the cluster core. RGB stars in the cluster's outskirts are bluer than the innermost RGB stars.

We adopted the isochrone defined by the cluster's overall physical properties as our benchmark and carefully investigated the magnitude spread of the SGB stars as a function of radius. The resulting $2 \mathrm{D}$ probability distribution displays two clear peaks between $R=600^{\prime \prime}$ and $R=900^{\prime \prime}$, and between $R=400^{\prime \prime}$ and $R=500^{\prime \prime}$. We followed a similar approach in our analysis of the cluster's RGB stars. The corresponding probability distribution shows a continuous ridge from the cluster's outer edge to $R \sim 500^{\prime \prime}$, followed by a peak between $R=200^{\prime \prime}$ and $R=400^{\prime \prime}$. For both SGB stars and RGB stars, the stars located at the largest 
radii are clearly different from the innermost sample stars.

We used a Monte Carlo method to estimate the contamination due to line-of-sight projection as a function of radius and conclude that the innermost radial ranges are, in fact, seriously contaminated by projected peripheral stars. This explains the larger magnitude (color) dispersion of the innermost SGB (RGB) stars compared with their counterparts at larger radii. In fact, if we could properly reconstruct the 3D distribution of the observed stars, the inner stellar sample would most likely be more clearly different from the outer sample.

We use different models in our attempts at explaining the observations, including dispersions in helium abundance, $[\alpha / \mathrm{Fe}]$, metallicity, and age. The most promising explanation is that the observed SGB and RGB stars are characterized by variations in helium and metal abundance, with the peripheral stars being both more helium-poor $(\mathrm{Y}=0.25)$ and more metal-poor $(Z=0.0033)$, while the more centrally located stars are likely both more helium-rich $(\mathrm{Y}=0.28)$ and more metal-rich $(Z=0.0051)$. The effects of $[\alpha / \mathrm{Fe}]$ variations are negligible; our photometric data are insufficiently sensitive to trace any such differences. The helium-rich, metal-rich sample is also consistent with a relatively younger isochrone, with age of $12.25 \mathrm{Gyr}$, while the helium-poor, metal-poor sample follows a 12.75 Gyr isochrone. An age dispersion of $\sim 0.5$ Gyr is required to best match the morphologies of both the SGB and RGB stars. In this context, the helium-abundance and metallicity dispersion invoked to explain the majority of the broadening of the CMD features would have originated from contamination by first-generation stars. We generate a series of simulated CMDs covering both the SGB and RGB stars, and use a $\chi^{2}$-minimization method to quantify the best-fitting first-generation stellar fraction. The result shows a clear, increasing trend from the innermost region to the cluster outskirts. This indicates that only a very small fraction of first-generation stars is contained in the cluster core, while the stellar population in the outskirts is close to a simple stellar population. The $\chi^{2}$-minimization results also show excellent agreement for both the SGB and RGB stars, both as regards the trend and the actual values, at least for $f_{\mathrm{fg}}$ at $R \in\left[400^{\prime \prime}, 600^{\prime \prime}\right]$, which strongly indicates that the SGB and RGB stars share the same stellar population composition.

Based on the analysis presented in this article, we thus confirm that 47 Tuc is composed of more than one stellar population. The most straightforward interpretation of the origin of a second stellar generation is that it is the remnant of the first stellar generation with enhanced helium abundance (from $\mathrm{Y}=0.25$ to $\mathrm{Y}=0.28$ ), as well as enhanced metallicity (from $Z=0.0033$ to $Z=0.0051$ ). 


\section{Acknowledgements}

The analysis in this article is based on observations made with the VISTA telescope at the European Southern Observatory under program ID 179.B-2003. We thank the team responsible for the UK's VISTA Data Flow System - comprising the VISTA pipeline at the Cambridge Astronomy Survey Unit (CASU) and the VISTA Science Archive at the Wide Field Astronomy Unit (Edinburgh; WFAU) - for providing calibrated data products, supported by the UK's Science and Technology Facilities Council. Partial financial support for this work was provided by the National Natural Science Foundation of China (NSFC) through grants 11073001 and 11373010 (RdG, CL). RG is a Pegasus postdoctoral fellow supported by the Fonds Wetenschappelijk Onderzoek (FWO), Flanders. B-QF is the recipient of a John Stocker Postdoctoral Fellowship from the Science and Industry Research

Fund (Australia). AEP acknowledges partial support from CONICET (Argentina) and the Agencia Nacional de Promoción Científica y Tecnológica (ANPCyT).

\section{REFERENCES}

Anderson, J., Piotto, G., King, I. R., \& Guhathakurta, P. 2009, ApJL, 607, L58

Arnaboldi, M., Rejkuba, M., Retzlaff, J., et al. 2013, The Messenger, 154, 18

Avni, Y. 1976, ApJ, 210, 642

Bekki, K. 2011, MNRAS, 412, 2241

Bastian, N., \& de Mink, S. E. 2009, MNRAS, 398, L11

Bastian, N., Cabrera-Ziri, I., Davies, B., \& Larsen, S. S. 2013, MNRAS, 436, 2852

Bonatto, C., Campos, F., \& Kepler, S. O., 2013, MNRAS, 435, 263

Boyer, M. L., van Loon, J. Th., McDonald, I., et al. 2010, ApJL, 711, 99

Brown, J. A., \& Wallerstein, G. 1992, AJ, 104, 1818

Carretta, E., \& Gratton, R. G. 1997, A\&AS, 121, 95

Carretta, E., Gratton, R. G., Bragaglia, A., Bonifacio, P., \& Pasquini, L. 2004, A\&A, 416, 925

Carretta, E., Bragaglia, A., Gratton, R. G., et al. 2009, A\&A, 505, 117 
Cassisi, S., Castellani, V., Degl'Innocenti, S., Salaris, M., \& Weiss, A. 1999, A\&AS, 134, 103

Cioni, M.-R. L., Clementini, G., Girardi, L., et al. 2011, A\&A, 527, A116

Cordero, M. J., Pilachowski, C. A., Johnson, C. I., et al. 2014, ApJ, 780, 94

Cross, N. J. G., Collins, R. S., Mann, R. G., et al. 2012, A\&A, 548A, 119

D’Antona, F., Gratton, R., \& Chieffi, A. 1983, Mem. Soc. Astron. It., 54, 173

Decressin, T., Meynet, G., Charbonnel, C., Prantzos, N., \& Ekstrom, S. 2007, A\&A, 464, 1029

Decressin, T., Baumgardt, H., \& Kroupa, P. 2008, A\&A, 492, 101

de Grijs, R., Johnson, R. A., Gilmore, G. F., \& Frayn, C. M. 2002a, MNRAS, 331, 228

de Grijs, R., Gilmore, G. F., Johnson, R. A., \& Mackey, A. D. 2002b, MNRAS, 337, 597

de Grijs, R., Gilmore, G. F., Mackey, A. D., Wilkinson, M. I., Beaulieu, S. F., Johnson, R. A., \& Santiago, B. X. 2002, MNRAS, 337, 597

de Grijs, R., Li, C., Zheng Y., et al. 2013, ApJ, 765, 4

de Mink, S. E., Pols, O. R., Langer, N., \& Izzard, R. G. 2009, A\&A, 507, L1

D’Ercole, A., Vesperini, E., D’Antona, F., McMillan, S., \& Recchi, S. 2008, MNRAS, 391, 825

Di Criscienzo, M., Venture, P., D’Antona, F., Milone, A., \& Piotto, G. 2010, MNRAS, 408, 999

Dotter, A., Sarajedini, A., Anderson, J., et al. 2010, ApJ, 708, 698

Ferraro, F. R., Dalessandro, E., Mucciarelli, A., et al. 2009, Natur, 462, 26

Girardi, L., Bressan, A., Bertelli, G., \& Chiosi, C. 2000, A\&AS, 141, 371

Girardi, L., Eggenberger, P., \& Andrea, M. 2011, MNRAS, 412, L103

Goldsbury, R., Richer, H. B., Anderson, J., et al. 2010, AJ, 140, 1830

Goudfrooij, P., Puzia, T. H., Chandar, R., \& Kozhurina-Platais, V. 2011, ApJ, 737, 4

Gratton, R. G., Bragaglia, A., Carretta, E., et al. 2003, A\&A, 408, 529 
Harris, W. E. 1996, AJ, 112, 1487

Harris, W. E. 2010, A New Catalog of Globular Clusters in the Milky Way (arXiv:1012.3224)

Irwin, M. J., Lewis, J., Hodgkin, S., et al. 2004, in SPIE Conf. Ser. 5493, eds. P. J. Quinn, \& A. Bridger, 411

Koch, A., \& McWillian, A., 2008, 135, 1551

Lane, R. R., Küpper, A. H. W., \& Heggie, D. C. 2012, MNRAS, 423, 2845

Langer, G. E., Hoffman, R., \& Sneden, C. 1993, PASP, 105, 301

Lee, L., Kang, Y., Lee, J., \& Lee, Y. 2009, Natur, 462, 480

Li, C., de Grijs, R., \& Deng, L. 2013, MNRAS, 436, 1497

Li, C., de Grijs, R., \& Deng, L. 2014, ApJ, 784, 157

Li, Z., Mao, C., Li, C., \& Zhang, Q. 2012, ApJL, 761, L22

Mackey, A. D., \& Broby Nielsen, P. 2007, MNRAS, 379, 151

Mackey, A. D., Broby Nielsen, P., Ferguson, A. M. N., \& Richardson, J. C. 2008, ApJL, 681, L17

Mackey, A. D., Da Costa, G. S., Ferguson, A. M. N., \& Yong, D. 2013, ApJ, 762, 65

Marín-Franch, A., Aparicio, A., Piotto, G., et al. 2009, ApJ, 694, 1498

Marino, A. F., Sneden, C., Kraft, R. P., et al. 2011, A\&A, 532, 8

Marino, A. F., Milone, A. P., Sneden, C., et al. 2012, A\&A, 541, 15

McDonald, I., Boyer, M., van Loon, J. Th., et al. 2011, ApJS, 193, 23

McLaughlin, D. E., Anderson, J., Meylan, G., et al. 2006, ApJS, 166, 249

McWillian, A., \& Bernstein, R. A. 2008, ApJ, 684, 326

Milone, A. P., Bedin, L. R., Piotto, G., et al. 2008, ApJ, 673, 241

Milone, A. P., Marino, A. F., Piotto, G., Bedin, L. R., Anderson, J., Aparicio, A., Cassisi, S., \& Rich, R. M. 2012, ApJ, 745, 27

Milone, A. P., Piotto, G., Bedin, L. R., et al. 2011, A\&A, 540, A16 
Milone, A. P., Piotto, G., Bedin, L. R., et al. 2012, ApJ, 744, 58

Momany, Y., Saviane, I., Smette, A., et al. 2012, A\&A, 537, 2

Monelli, M., Milone, A. P., Stetson, P. B., et al. 2013, MNRAS, 431, 2126

Nataf, D. M., Gould, A., Pinsonneault, M. H., \& Stetson, P. B. 2011, ApJ, 736, 94

Norris, J., \& Freeman, K. C. 1979, ApJ, 230, 179

Norris, J., \& Freeman, K. C. 1982, MNRAS, 254, 143

Norris, J., Freeman, K. C., Da Costa, G. S. 1984, ApJ, 277, 615

Piotto, G., Villanova, S., Bedin, L. R., et al. 2005, ApJ, 621, 777

Piotto, G., Bedin, L. R., et al. 2007, ApJL, 661, L53

Piotto, G., Milone, A. P., Marino, A. F., Bedin, L. R., Anderson, J., Jerjen, H., Bellini, A., \& Cassisi, S. 2013, ApJ, 775, 15

Platais, I., Melo, C., Quinn, S. N., et al. 2012, ApJL, 751, L8

Ramírez, S. V., \& Cohen, J. G. 2002, AJ, 123, 3277

Qing, B., \& Sneden, C. 2010, ApJ, 140, 1694

Renzini, A. 1983, Mem. Soc. Astron. It., 8, 61

Rubele, S., Kerber, L., \& Girardi, L. 2010, MNRAS, 403, 1156

Rubele, S., Girardi, L., Kozhurina-Platais, V., et al. 2013, MNRAS, 430, 2774

Salasnich, B., Girardi, L., Weiss, A., \& Chiosi, C. 2000, A\&A, 361, 1023

Salaris, M., Held, E. V., Otolani, S., et al. 2007, A\&A, 243, 253

Schlafly, E. F., \& Finkbeiner, D. P. 2011, ApJ, 737, 103

Schlegel, D. J., Finkbeiner, D. P., \& Davis, M. 1998, ApJ, 500, 525

Sollima, A., Ferraro, F. R., Bellazzini, M., et al. 2007, ApJ, 550, 65

Stetson, P. B. 1987, PASP, 99, 191 
Trager, S. C., Djorgovski, S. G., \& King, I. R. 1993, in ASP Conf. Ser. 50, Structure and Dynamics of Globular Clusters, ed. S. G. Djorgovski \& G. Meylan (San Francisco: ASP), 347

Valcarce, A. A. R., Catelan, M., \& Sweigart, A. V. 2012, A\&A, 547, 5

Vandenberg, D. A., Brogaard, K., Leaman, R., \& Casagrande, L. 2013, ApJ, 775, 134

Ventura, P., \& D'Antona, F. 2009, A\&A, 499, 835

Ventura, P., Di Criscienzo, M., D'Antona, F., et al. 2014, MNRAS, 437, 3274

Vesperini, E., McMillan, S. L. W., D’Antona, F., \& D’Ercole, A. 2013, MNRAS, 429, 1913

Wall J. V., 1996, QJRAS, 37, 519

Yang, W., Bi, S., Meng, X., \& Liu, L. 2013, ApJ, 776, 112

Zoccali, M., Renzini, A., Ortolani, S., et al. 2001, ApJ, 553, 733 

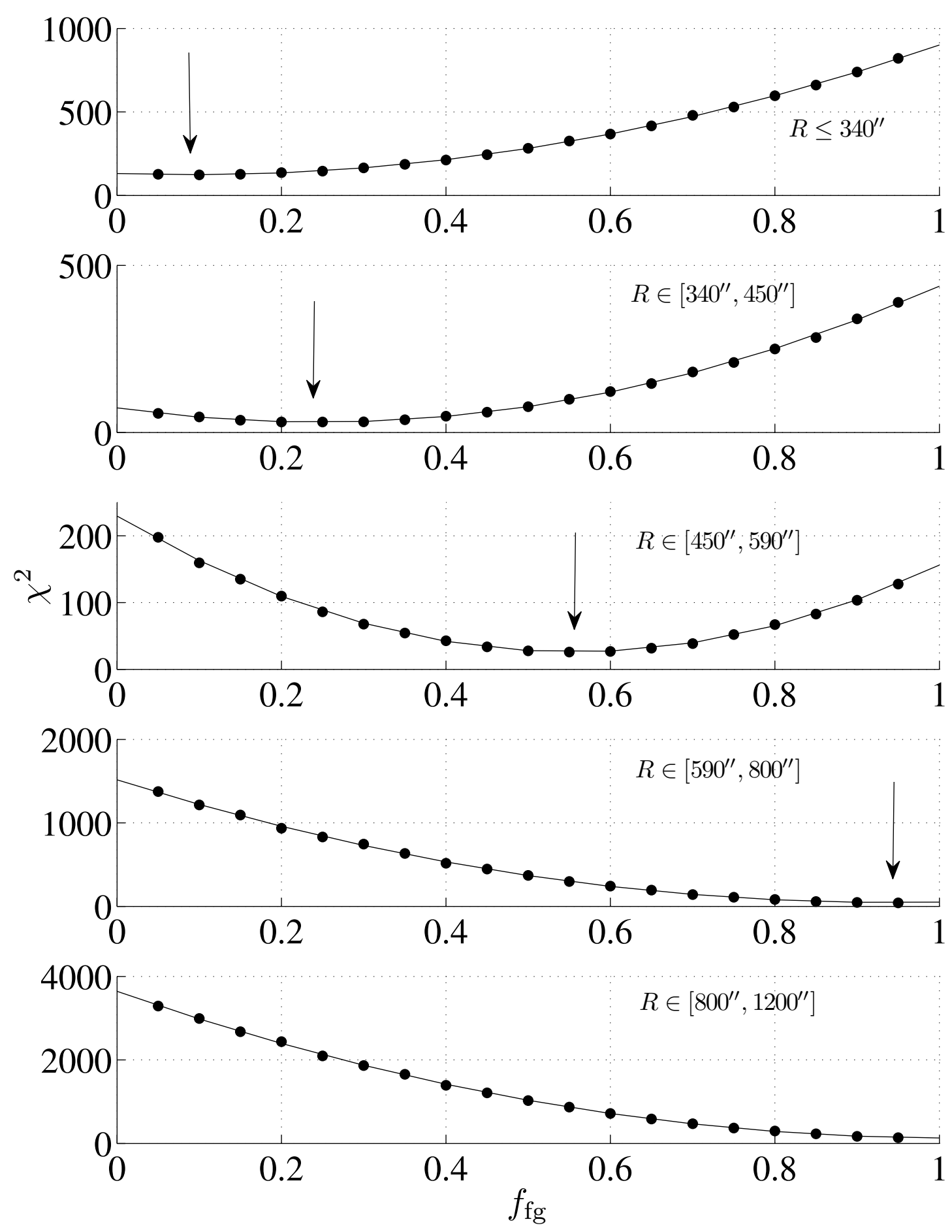

Fig. 18. $-\chi^{2}$ as a function of $f_{\mathrm{fg}}$ for the SGB stars. From top to bottom, the panels indicate the results from the innermost region to the cluster outskirts. The curve represents the best-fitting parabolic function; the arrows indicate the minimum $\chi^{2}$ values and, hence, the best-fitting $f_{\mathrm{fg}}$. The bottom panel exhibits a best-fitting $f_{\mathrm{fg}}$ of unity. 

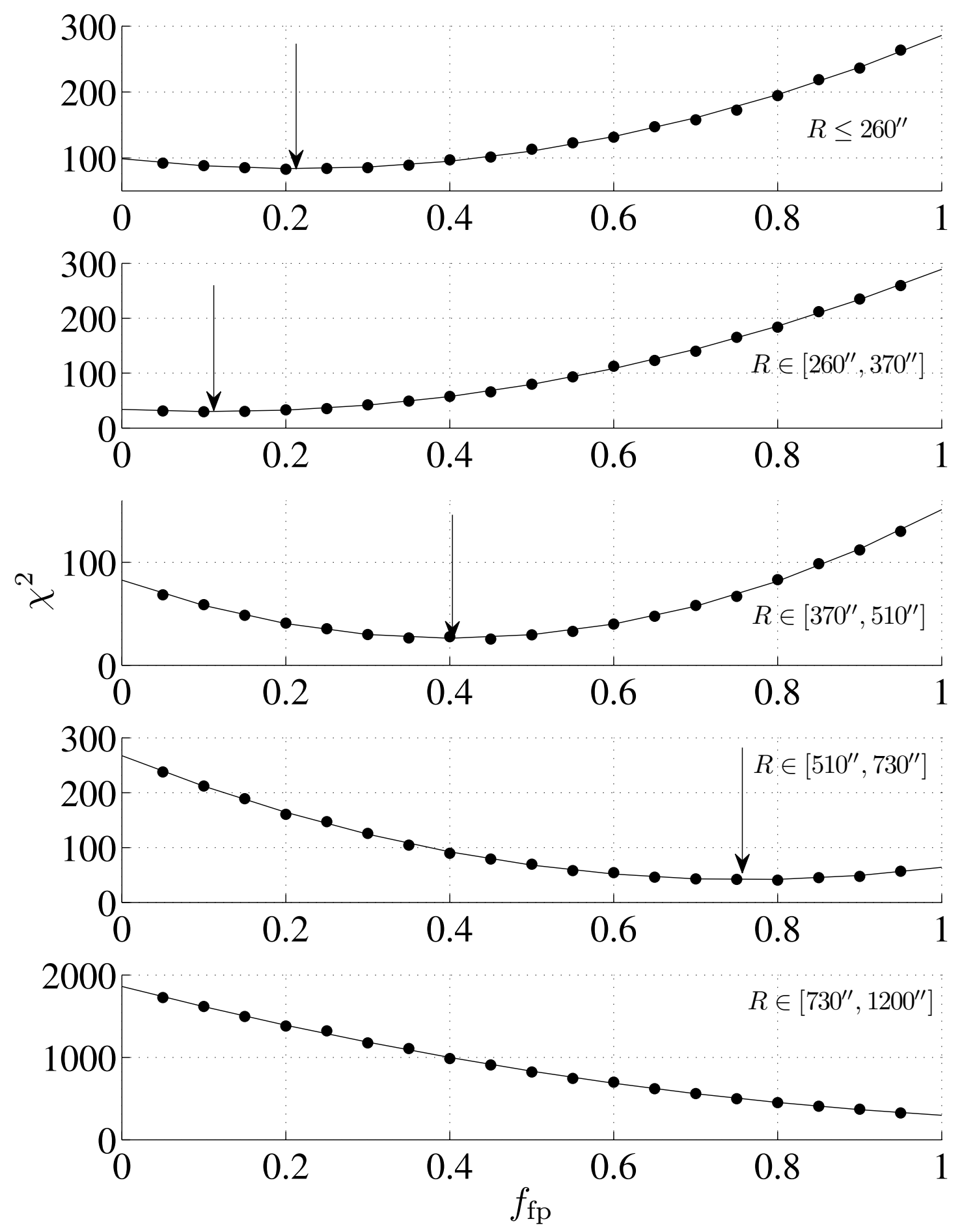

Fig. 19.- As Figure 18, but for the RGB stars. 


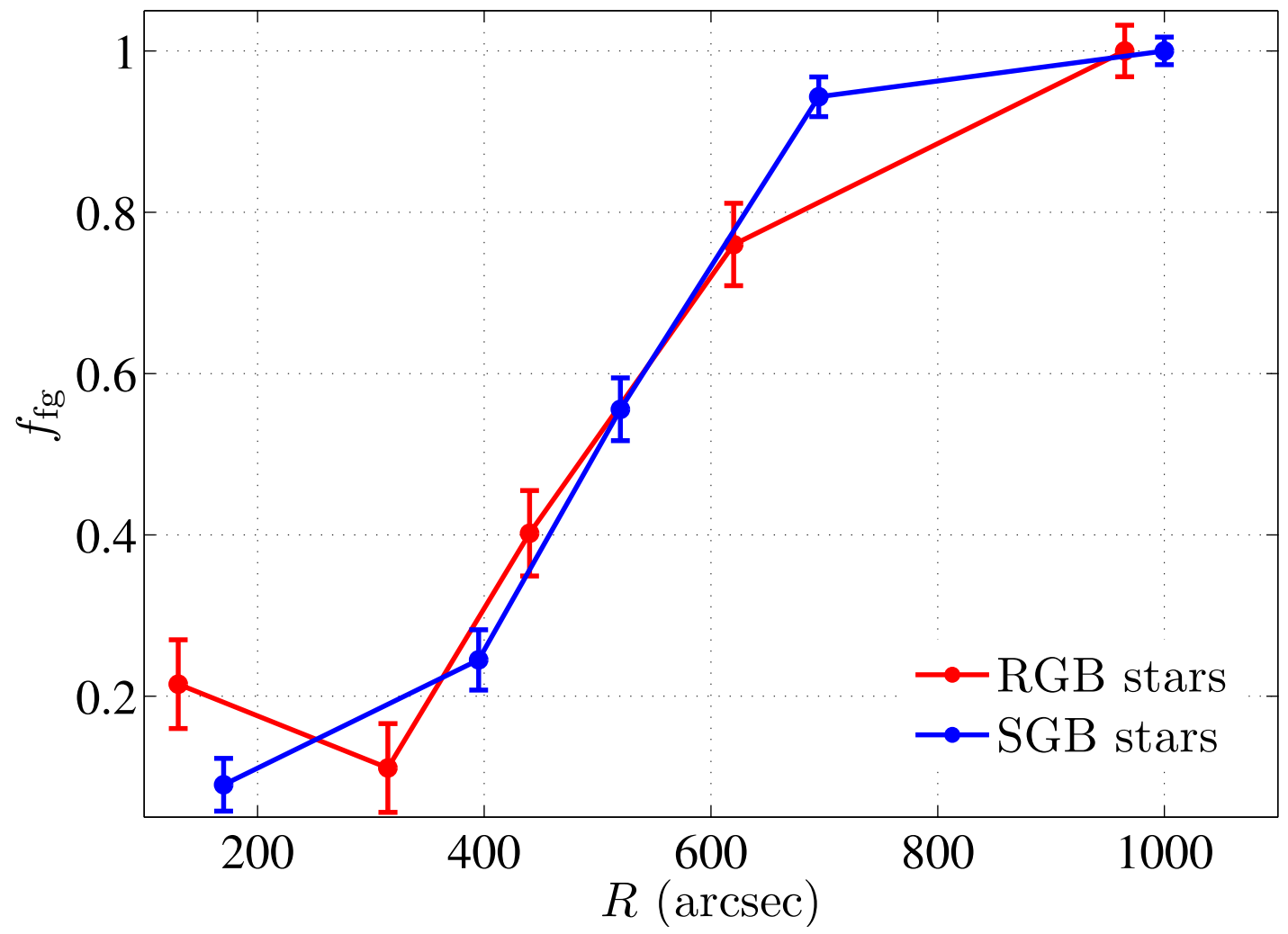

Fig. 20.- Best-fitting $f_{\text {fg }}$ fraction as a function of radius for SGB stars (blue) and RGB stars (red). The error bars represent the $1 \sigma$ statistical uncertainties, which are all less than $5.5 \%$. 